PHYSICAL REVIEW D 94, 094507 (2016)

\title{
SU(2) gauge theory with two fundamental flavors: A minimal template for model building
}

\author{
Rudy Arthur, ${ }^{1, *}$ Vincent Drach, ${ }^{2, \dagger}$ Martin Hansen, ${ }^{1, *}$ Ari Hietanen, ${ }^{1, \S}$ Claudio Pica, ${ }^{1, \|}$ and Francesco Sannino ${ }^{1, \pi}$ \\ ${ }^{1} C P^{3}$-Origins \& the Danish IAS, University of Southern Denmark, \\ Campusvej 55, DK-5230 Odense M, Denmark \\ ${ }^{2}$ Theoretical Physics Department, CERN, Geneva CH-1211, Switzerland
}

(Received 23 March 2016; published 29 November 2016)

\begin{abstract}
We investigate the continuum spectrum of the SU(2) gauge theory with $N_{f}=2$ flavors of fermions in the fundamental representation. This model provides a minimal template which is ideal for a wide class of Standard Model extensions featuring novel strong dynamics that range from composite (Goldstone) Higgs theories to several intriguing types of dark matter candidates, such as the strongly interacting massive particles (SIMPs). We improve our previous lattice analysis [1] by adding more data at light quark masses, at two additional lattice spacings, by determining the lattice cutoff via a Wilson flow measure of the $w_{0}$ parameter, and by measuring the relevant renormalization constants nonperturbatively in the regularizationinvariant momentum (RI'-MOM) scheme. Our result for the lightest isovector state in the vector channel, in units of the pseudoscalar decay constant, is $m_{V} / F_{\mathrm{PS}} \sim 13.1(2.2)$ (combining statistical and systematic errors). For the axial channel our result is $m_{A} / F_{\mathrm{PS}} \sim 14.5$ (3.6), which however does include a similarly sized additional systematic error due to residual excited-states contamination. In the context of the composite (Goldstone) Higgs models, our result for the spin-one resonances are $m_{V}>3.2(5) \mathrm{TeV}$ and $m_{A}>3.6(9) \mathrm{TeV}$, which are above the current LHC constraints. In the context of dark matter models, for the SIMP case our results indicate the occurrence of a compressed spectrum at the required large dark pion mass, which implies the need to include the effects of spin-one resonances in phenomenological estimates.
\end{abstract}

DOI: 10.1103/PhysRevD.94.094507

\section{INTRODUCTION}

New composite dynamics is often invoked to construct extensions of the Standard Model (SM) physics that can address one or several of the SM's shortcomings.

For example, composite extensions have been suggested to replace the SM Higgs sector, to suggest natural dark matter (DM) candidates and, more recently, to explain [2-4] the observed tantalizing excess in the diphoton decay channel or earlier diboson excesses $[5,6]$ recorded by the CMS and ATLAS experiments [7-9]. Time-honored classes of fundamental electroweak composite dynamics are technicolor (TC) $[10,11]$ and composite Goldstone Higgs models $[12,13]$.

In TC models the Higgs boson is the lightest scalar excitation of the fermion condensate responsible for electroweak (EW) symmetry breaking [14-18]. The

\footnotetext{
*arthur@cp3-origins.net

†vincent.drach@cern.ch

"hansen@cp3-origins.net

\$hietanen@cp3-origins.net

pica@cp3-origins.net

sannino@cp3-origins.net
}

Published by the American Physical Society under the terms of the Creative Commons Attribution 4.0 International license. Further distribution of this work must maintain attribution to the author(s) and the published article's title, journal citation, and DOI. physical technicolor Higgs mass can be light due to near-conformal dynamics [14,19] and the interplay between the TC sector and the SM fermions and electroweak gauge bosons [20].

In composite Goldstone Higgs models [12,13], the new sector has an underlying fundamental dynamics with a larger global symmetry group than the one strictly needed to break the EW symmetry. In this case the Higgs state can be identified with one of the additional Goldstone bosons (GBs), and it is therefore naturally light. However, to break the EW symmetry, typically radiative corrections are not enough and yet another sector is required to induce the correct vacuum alignment for the EW gauge bosons and for the Higgs to acquire the observed mass.

The underlying fundamental theory studied here constitutes the ultra-minimal composite template for any natural UV completion that simultaneously embodies both the TC and composite Goldstone Higgs models [21-24]. It is also well known that fermion mass generation constitutes a challenge for any composite dynamic extension. For the present theory an extension that makes use of chiral gauge theories [25-27] has been put forward recently in Ref. [28]. The constructions yield distinctive experimental signatures and can be used universally for both types of model building.

Novel composite dynamics has also been advocated to construct natural candidates for DM stemming from a composite EW sector. In fact, several asymmetric DM 
candidates were put forward which are stable baryons in TC models [29,30] or Goldstone bosons of a new strong sector [22,31-33].

Another interesting class of DM models, unrelated to the composite EW scenario, was recently revived in Ref. [34]. Here an alternative mechanism [35,36] is employed for achieving the observed DM relic density. It uses $3 \rightarrow 2$ number-changing processes that should occur in the dark sector involving strongly interacting massive particles (SIMPs). Compared to the weakly interacting massive particle paradigm, where the dark matter particles typically are expected to be around the $\mathrm{TeV}$ scale, this model can yield dark matter particles with masses of a few $100 \mathrm{MeV}$. In Refs. [37,38] a realization of the SIMP paradigm was introduced in terms of composite theories for which the model investigated here again provides the minimal template. In this realization, the pions constitute the dark matter particles and the topological Wess-Zumino-Witten (WZW) term [39-41] introduces a five-point pion interaction, making it an ideal candidate for the $3 \rightarrow 2$ annihilation process. The most minimal realization of this breaking pattern comes indeed from the underlying $\mathrm{Sp}(2)=\mathrm{SU}(2)$ gauge group [but it can be generalized to any $\operatorname{Sp}\left(\mathrm{N}_{c}\right)$ gauge group]. The first consistent investigation of the phenomenological viability of this construction, that properly took into account important next-to-next-to-leading-order (NNLO) corrections via chiral perturbation theory, appeared in Ref. [38]. There it was shown that higherorder corrections substantially increase the tension with phenomenological constraints. Because the energy scale of the SIMP is very light, it is especially relevant to know at which energy scale dark spin-one resonances will appear, or more generally to understand its spectroscopy [42]. Furthermore the new states will modify the scattering at higher energies introducing possible interesting resonant behaviors [43] and, as it is the case for ordinary QCD, will have an impact on a number of dark-sector-induced physical observables.

In this work we investigate the $\mathrm{SU}(2)$ gauge theory with $N_{f}=2$ flavors of Dirac fermions in the fundamental representation. One important feature of this minimal SU(2) template model is that, due to the pseudoreality of the fundamental representation, the flavor symmetry is upgraded to an $\mathrm{SU}(4)$ [locally isomorphic to $\mathrm{SO}(6)$ ] symmetry which is expected to break spontaneously to $\mathrm{Sp}$ (4) [locally isomorphic to $\mathrm{SO}(5)$ ], thus leading to five Goldstone bosons.

The theory has previously been studied on the lattice, and in particular, it has been shown that the expected pattern of spontaneous chiral symmetry breaking is realized [44]. A first estimate, affected by large systematic errors, of the masses of the vector and axial-vector mesons, in units of the pseudoscalar meson decay constant, have been obtained in Ref. [1]. The scattering properties of the Goldstone bosons of the theory have also been considered [45], and the model has furthermore been investigated in the context of possible DM candidates related to the EW scale $[46,47]$. Other groups have also investigated the spectrum of this model on the lattice $[48,49]$ concluding that chiral symmetry is broken, although no continuum extrapolation was attempted as the focus of both works was on the comparison with the six-flavor theory to understand the approach to the conformal window in $S U(2)$ gauge theories.

Here we extend our previous analyses by improving our control on the systematics. Our simulations achieve smaller fermion masses, include two additional lattice spacings, and we also perform a precise determination of the lattice spacings used. Finally we determine the relevant renormalization constants nonperturbatively.

The paper is organized as follows. We first describe the lattice setup in Sec. II and the procedure to set the lattice spacing through the Wilson flow observable $w_{0}$ in Sec. III. In Sec. IV we discuss the calculation of the renormalization constants using the RI'-MOM scheme. Finally we provide in Sec. V an improved estimation of the spectrum of the theory.

\section{LATTICE SETUP}

We simulate the SU(2) gauge theory with two Dirac fermions in the fundamental representation discretized using the (unimproved) Wilson action for two massdegenerate fermions $u, d$ and the Wilson plaquette action for the gauge field. The numerical simulations have been performed using an improved version of the HIREP code first described in Ref. [50]. The fermionic part of the action reads

$$
\begin{aligned}
S_{F}= & \sum_{x} \bar{\psi}(x)\left(4+a m_{0}\right) \psi(x) \\
& -\frac{1}{2} \sum_{x, \mu}\left(\bar{\psi}(x)\left(1-\gamma_{\mu}\right) U_{\mu}(x) \psi(x+\hat{\mu})\right. \\
& \left.+\bar{\psi}(x-\hat{\mu})\left(1+\gamma_{\mu}\right) U_{\mu}^{\dagger}(x) \psi(x)\right),
\end{aligned}
$$

where $U_{\mu}$ is the gauge field, $\psi$ is the doublet of $u$ and $d$ fermions, and $a m_{0}$ is the $2 \times 2$ diagonal mass matrix proportional to the identity.

Our simulations are performed at four values of the inverse lattice gauge coupling $\beta$, for various fermion masses and on several volumes. This is needed in order to perform the required extrapolations to the chiral limit and infinite volume and to give an estimate of the systematic errors stemming from such extrapolations. We detail the procedure used in the following sections.

The bare parameters of our simulations are summarized in Table I. We have extended our previously published data set considerably, in particular towards the chiral regime and by adding two additional lattice spacings at $\beta=1.8,2.3$. As we will discuss in more detail below, note that the lightest quark masses now reach, in some cases, the decay 
TABLE I. Parameters used in the simulations. Runs with * are used only to study finite-size effects. All the others runs are referred to in the text as "large-volume runs."

\begin{tabular}{ccc}
\hline \hline$\beta$ & Volume & $a m_{0}$ \\
\hline 1.8 & $16^{3} \times 32$ & $-1.00,-1.089,-1.12,-1.14,-1.15,-1.155^{*}$ \\
1.8 & $32^{3} \times 32$ & $-1.155-1.557$ \\
2.0 & $16^{3} \times 32$ & $-0.85,-0.9,-0.94,-0.945,-0.947^{*}$ \\
2.0 & $32^{4}$ & $-0.947,-0.949,-0 .-952,-0.957,-0.958$ \\
2.2 & $16^{3} \times 32$ & $-0.60,-0.65,-0.68-0.70,-0.72^{*},-0.75^{*}$ \\
2.2 & $24^{3} \times 32$ & $-0.75^{*}$ \\
2.2 & $32^{4}$ & $-0.72,-0.735,-0.75$ \\
2.2 & $48^{4}$ & -0.76 \\
2.3 & $32^{4}$ & $-0.575,-0.60,-0.625,-0.65,-0.675,-0.685$ \\
\hline \hline
\end{tabular}

threshold for the vector-meson resonance. The simulations in Table I denoted with an asterisk, are only used to study the systematic errors due to finite-size effects. The remaining runs will be referred to as "large-volume runs" in this paper. For all these lattices with $m_{\mathrm{PS}} L \geq 5$, we expect that systematic errors due to finite volume are of the order of $5-10 \%$ for the measured meson masses relevant in this work, based on the analysis presented in Ref. [1]. There it was found that, in particular the mass of the axial-vector state, as extracted in the current work, suffers from larger systematic effects of the order of $10 \%$.

For convenience, we define the following operators:

$$
\mathcal{O}_{\bar{u} d}^{(\Gamma)}(x)=\bar{u}(x) \Gamma d(x),
$$

where $\Gamma$ denotes any product of Dirac matrices.

We extract the meson masses from zero-momentum twopoint correlation functions

$$
f_{\Gamma}(t)=\sum_{\vec{x}}\left\langle\mathcal{O}_{\bar{u} d}^{(\Gamma)}(t, \vec{x})^{\dagger} \mathcal{O}_{\bar{u} d}^{(\Gamma)}(0)\right\rangle
$$

The quantities of interest in this study are the pseudoscalar $\Gamma=\gamma_{5}$, vector $\Gamma=\gamma_{k}(k=1,2,3)$, and axial-vector $\Gamma=\gamma_{0} \gamma_{5} \gamma_{k}$ mesons. We use $Z_{2} \times Z_{2}$ single time slice stochastic sources [51] to estimate the meson two-point correlators. From those, we define an effective mass $m_{(\Gamma)}^{\text {eff }}(t)$ as in Refs. [52,53] by the solution of the implicit equation:

$$
\frac{f_{\Gamma}(t-1)}{f_{\Gamma}(t)}=\frac{e^{-m_{(\Gamma)}^{\text {eff }}(t)(T-(t-1))}+e^{-m_{(\Gamma)}^{\text {eff }}(t)(t-1)}}{e^{-m_{(\Gamma)}^{\text {eff }}(t)(T-t)}+e^{-m_{(\Gamma)}^{\text {eff }}(t) t}},
$$

where $T$ is the lattice time extent. At large Euclidean time, $m_{(\Gamma)}^{\text {eff }}(t)$ approaches the value of the mass of the lightest state with the same quantum numbers as the operator $\mathcal{O}_{\bar{u} d}^{(\Gamma)}$. In the following, we will denote the pseudoscalar meson mass by $m_{\mathrm{PS}}$, and the isovector vector and axial-vector meson masses by $m_{\mathrm{V}}$ and $m_{\mathrm{A}}$ respectively.
In addition to the meson masses above, we will use in the present analysis two other quantities: the current quark mass $m_{\mathrm{PCAC}}$ and the Goldstone boson decay constant $F_{\mathrm{PS}}$. We define the quark mass through the partially conserved axial current (PCAC) relation

$$
m_{\mathrm{PCAC}}=\lim _{t \rightarrow \infty} \frac{1}{2} \frac{\partial_{t} f_{A P}(t)}{f_{\gamma_{5}}(t)}
$$

where

$$
f_{A P}(t)=\sum_{\vec{x}}\left\langle\mathcal{O}_{\bar{u} d}^{\left(\gamma_{0} \gamma_{5}\right)}(t, \vec{x})^{\dagger} \mathcal{O}_{\bar{u} d}^{\left(\gamma_{5}\right)}(0)\right\rangle
$$

The Goldstone boson decay constant can be calculated as

$$
F_{\mathrm{PS}}=\frac{2 m_{\mathrm{PCAC}}}{m_{\mathrm{PS}}^{2}} G_{\mathrm{PS}},
$$

where $G_{\mathrm{PS}}$ is obtained from the asymptotic form of $f_{\gamma_{5}}(t)$ at large $t$ :

$$
f_{\gamma_{5}}(t) \sim-\frac{G_{\mathrm{PS}}^{2}}{m_{\mathrm{PS}}} \exp \left[-m_{\mathrm{PS}} t\right] .
$$

On a lattice of finite temporal extent, we use the same definitions as in Refs. [52,53].

The (bare) values in lattice units for $m_{\mathrm{PCAC}}, m_{\mathrm{PS}}, F_{\mathrm{PS}}$, $m_{\mathrm{V}}$ and $m_{\mathrm{A}}$ corresponding to the large-volume lattices considered in this paper are reported in Table VII in Appendix A.

To convert the lattice quantities to physical units, we determine the lattice spacing for our simulations and the appropriate nonperturbative renormalization constants.

It is well known that for Wilson fermions, the pseudoscalar decay constant renormalizes multiplicatively with the scale-independent renormalization constant $Z_{A}$ and that the bare PCAC mass renormalizes with the ratio $Z_{A} / Z_{P}\left(\mu^{2}\right)$.

The lattice spacing, in a generic composite model, is fixed by the requirement that the renormalized Goldstone boson decay constant has a given value specified for the physical model considered. For example in the case of composite dynamics at the electroweak scale, a value of $246 \mathrm{GeV}$ yields the correct mass for the electroweak gauge bosons. For the more general fundamental composite Goldstone Higgs scenario described in Ref. [24] the scale is still set by the same requirement, but the constraint on the renormalized Goldstone boson decay constant now reads $F_{\mathrm{PS}} \sin (\theta)=246 \mathrm{GeV}$. The actual value of the parameter $\theta$ in this model depends on the electroweak gauge boson corrections, the top corrections as well as the effects of other possible sources of explicit breaking of the initial $\mathrm{SU}(4)$ symmetry. The technicolor limit is recovered for $\theta=\pi / 2$ while the composite pseudo-GB Higgs case 
corresponds to small, but nonvanishing $\theta$. Any other value of $\theta$ is also allowed and the resulting model thus interpolates between these two limits. For the details we refer to Ref. [24].

Another case of immediate interest is the SIMPlest composite model [37] for DM where, as shown in Ref. [38], it is important to control the underlying dynamics. By stretching chiral perturbation theory to its limit of validity, the interesting phenomenological values for the pion decay constant would be as low as $10 \mathrm{MeV}$ with pion masses of the order of $100 \mathrm{MeV}$. Besides the rescaling of the pion decay constant, another major difference, when compared to composite dynamics at the electroweak scale, resides in the fact that the SIMP requires quite massive pions.

For definiteness, below we present our results in units of the EW scale with $\sin (\theta)=1$ but the dependence on $\theta$ can be reinstated when needed. At the end we will also comment on the results for the SIMPlest case.

\section{SCALE SETTING}

Following Ref. [54], we consider the following "Wilson flow" equation for the gauge fields:

$$
\begin{aligned}
\frac{d}{d t} V_{t}(x, \mu) & =-g_{0}^{2}\left\{\partial_{x, \mu} S_{G}\left(V_{t}\right)\right\} V_{t}(x, \mu) \quad \text { with } \\
V_{t=0}(x, \mu) & =U(x, \mu),
\end{aligned}
$$

where $t$ denotes the fictitious flow "time," $U(x, \mu)$ are the gauge links, and $S_{G}$ is the plaquette gauge action. One important property is that correlation functions at flow time $t>0$ are finite, when the four-dimensional theory is renormalized as usual, and the flow thus maps gauge fields into smooth, renormalized gauge fields [55]. Observables at nonzero flow time can, in particular, be used to define a scale, as shown in Ref. [54].

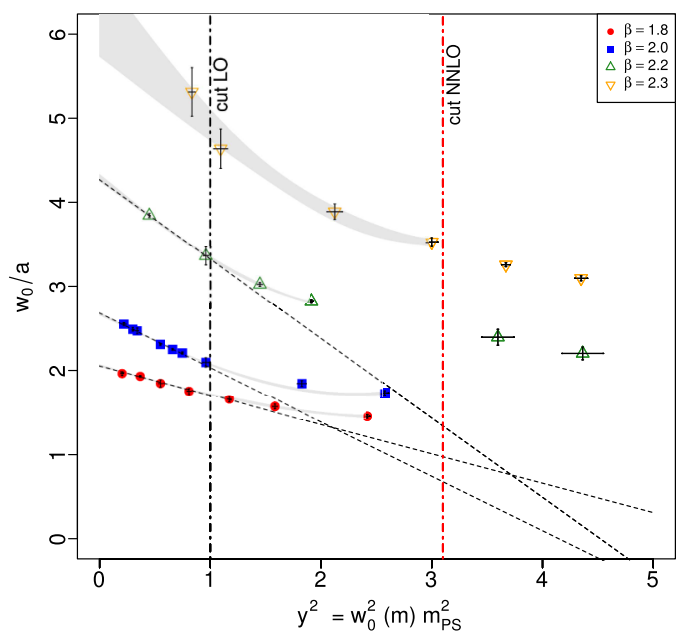

Two different scale-setting observables have been introduced in the literature, known as $t_{0}$ [54] and $w_{0}$ [56]. In terms of $E(t)$, the action density at flow time $t$, they are defined through the following equations:

$$
\begin{gathered}
\mathcal{E}(t)=t^{2} E(t), \quad \mathcal{E}\left(t_{0}\right)=\mathcal{E}_{\text {ref }}, \\
W(t)=t \frac{d}{d t} \mathcal{E}(t), \quad W\left(w_{0}^{2}\right)=W_{\text {ref }},
\end{gathered}
$$

where $\mathcal{E}_{\text {ref }}$ and $W_{\text {ref }}$ are two dimensionless reference values. In this work we will use $w_{0}$ to set the scale. The value of $w_{0}$ obtained for each quark mass needs to be extrapolated to the chiral limit to obtain a scale $w_{0}^{\chi}$ for each lattice spacing.

We investigated finite-volume errors in $w_{0}$ at the chosen reference value $W_{\text {ref }}$ by comparing two simulations performed on spacial sizes $L=16\left(m_{\mathrm{PS}} L \sim 5.1\right)$ and $L=32$ $\left(m_{\mathrm{PS}} L \sim 8.4\right)$ at bare parameters $m_{0}=-0.75$ and $\beta=2.2$. These values of the bare parameters were chosen to correspond to one of the lightest points in our data set, at a fine lattice spacing. The values of $w_{0}$ obtained are $w_{0}(L=16)=3.39(6) a$ and $w_{0}(L=32)=3.36(10) a$ which agree well within statistical errors, indicating that finite-volume effects for $w_{0}$ can be safely neglected for $m_{\mathrm{PS}} L>5$ within our numerical precision.

\section{A. Determination of $w_{0}^{\chi}$}

In Fig. 1 (left panel) we show our results for $w_{0} / a$ for the four lattice spacings considered in this study as a function of $y^{2}$, where $y=w_{0}\left(m_{\mathrm{PCAC}}\right) m_{\mathrm{PS}}$. Here the reference value chosen is $W_{\text {ref }}=1$. For all the points in Fig. 1 we have $m_{\mathrm{PS}} L>5.5$ and are thus safe from finite-volume effects.

In order to extrapolate to the chiral limit, we use the NNLO expansion in terms of $m_{\mathrm{PS}}^{2}$ which reads [57]

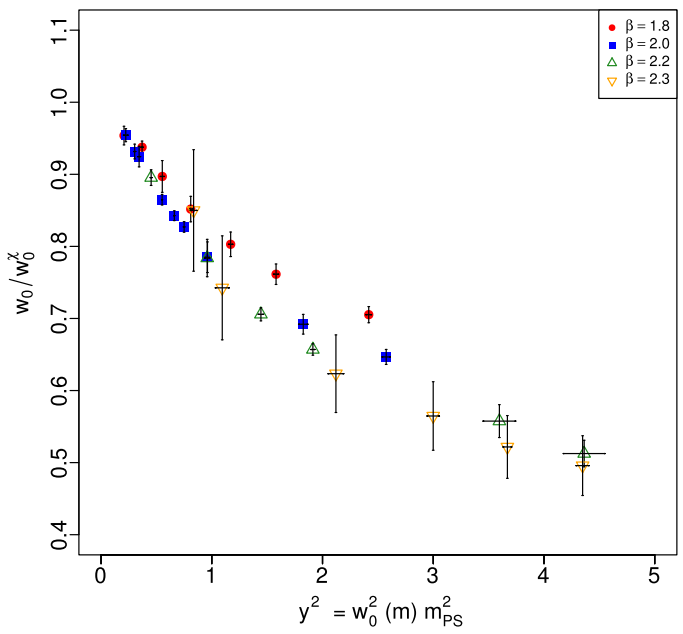

FIG. 1. Chiral behavior of $w_{0}$ as a function of $y^{2}$ in units of the lattice spacing (left panel) and in units of $w_{0}^{\chi}$ (right panel) for $W_{\text {ref }}=1$. The data at four lattice spacings are displayed. 
TABLE II. Summary of the NNLO fits for $w_{0} / a$ for each value of the lattice spacing. We chose $W_{\text {ref }}=1$ as a reference value.

\begin{tabular}{llccc}
\hline \hline$\beta$ & \multicolumn{1}{c}{$w_{0}^{\chi} / a$} & $A$ & $B$ & $\chi^{2} /$ ndof \\
\hline 1.8 & $2.066(16)$ & $-0.169(12)$ & $0.022(6)$ & $5.5 / 4$ \\
2.0 & $2.675(20)$ & $-0.223(10)$ & $0.036(4)$ & $14.8 / 6$ \\
2.2 & $4.311(49)$ & $-0.224(12)$ & $0.036(8)$ & $1.0 / 1$ \\
2.3 & $6.202(477)$ & $-0.205(39)$ & $0.018(9)$ & $2 / 1$ \\
\hline \hline
\end{tabular}

$w_{0}\left(m_{\mathrm{PS}}^{2}\right)=w_{0}^{\chi}\left(1+k_{1} \frac{m_{\mathrm{PS}}^{2}}{(4 \pi F)^{2}}+k_{2} \frac{m_{\mathrm{PS}}^{4}}{(4 \pi F)^{4}} \log \frac{m_{\mathrm{PS}}^{2}}{\mu^{2}}\right)$,

where $F$ is the pseudoscalar decay constant and $k_{1}, k_{2}$ are dimensionless low-energy constants. Note that the chiral logarithm enters only at NNLO. In practice we fitted our data at each $\beta$ with the following ansatz:

$$
w_{0}\left(m_{\mathrm{PS}}^{2}\right)=w_{0}^{\chi}\left(1+A y^{2}+B y^{4} \log y^{2}\right),
$$

where $A, B$ and $w_{0}^{\chi}$ are free parameters with the choice $w_{0}^{\chi} \mu=1$.

The fit is performed for each of the four $\beta$ values independently and the gray bands indicate the $1 \sigma$ error regions. The best-fit parameters and their statistical errors are reported in Table II. In the left panel of Fig. 1, the red dotted vertical line indicates the upper limit of the $y^{2}$ region used in the NNLO fit.

For three of our data sets we have also performed a fit to the NLO expression. The black vertical dotted line indicates the upper limit of the $y^{2}$ region included in the NLO fit. Due to lack of data, we cannot perform this fit for $\beta=2.3$. For the three remaining lattice spacings available, the results of the NLO and NNLO fits agree well within uncertainties.

In the right panel of Fig. 1 we show $w_{0} / w_{0}^{\chi}$ for all four lattice spacings. The deviation from a universal curve of such a quantity is a measure of lattice discretization errors. As can be seen, these are small in the $w_{0}$ observable for our three finest lattice spacings. The same conclusion can also be reached by looking at the dimensionless coefficients $A$ and $B$ as determined from the fits, given in Table II.

\section{NONPERTURBATIVE RENORMALIZATION CONSTANTS}

\section{A. RI'-MOM scheme}

In this section we describe the method used to determine the nonperturbative renormalization constants of the isovector vector $(\mathrm{V})$, axial $(\mathrm{A})$, and pseudoscalar $(\mathrm{P})$ bilinear operators. They are needed for the renormalization of the pseudoscalar decay constant $F_{\mathrm{PS}}$ and of the quark mass

$m_{\text {PCAC. }}$.
We use the RI'-MOM scheme as in Ref. [58]. We define the bilinear operators

$$
\begin{aligned}
O_{\Gamma}(x) & =\bar{\psi}(x) \tau^{3} \Gamma \psi(x), \quad \text { with } \\
\Gamma \in\{P, V, A, S\} & \equiv\left\{\gamma_{5}, \gamma_{\mu}, \gamma_{5} \gamma_{\mu}, 1\right\},
\end{aligned}
$$

and the fermion propagator

$$
\begin{aligned}
S(x, y) & =\langle\psi(x) \bar{\psi}(y)\rangle, \quad \text { and } \\
S(p) & =\sum_{p} e^{i p(x-y)} S(x, y) .
\end{aligned}
$$

Note that we have not explicitly written spin and color indices. We also define the Green's function

$$
G_{\Gamma}(p)=\left\langle\psi(p) O_{\Gamma}(p) \bar{\psi}(p)\right\rangle
$$

and we will denote the corresponding vertex function by

$$
\Pi_{\Gamma}(p)=S(p)^{-1} G_{\Gamma}(p) S(p)^{-1},
$$

where $S^{-1}(p)$ is the inverse propagator in spin and color space. The RI'-MOM scheme [58] is then defined by imposing the conditions that in the chiral limit and at a given scale $p^{2}=\mu^{2}$, the inverse propagator and amputated Green's function $\Pi_{\Gamma}(p)$ satisfy the following equations:

$$
\begin{gathered}
\left.Z_{q}^{-1} \frac{-i}{4 N_{c}} \operatorname{tr}\left[\frac{\gamma_{\mu} \sin \left(a p_{\mu}\right)}{\sin ^{2}\left(a p_{\mu}\right)} S^{-1}(p)\right]\right|_{p^{2}=\mu^{2}}=1, \quad \text { and } \\
\left.Z_{q}^{-1} Z_{\Gamma} \frac{1}{4 N_{c}} \operatorname{tr}\left[P_{\Gamma} \Pi_{\Gamma}(p)\right]\right|_{p^{2}=\mu^{2}}=1,
\end{gathered}
$$

where the trace is over spin and color indices and the projectors $P_{\Gamma}$ are defined as

$$
P_{\Gamma} \in\left\{P_{P}, P_{V}, P_{A}, P_{S}\right\} \equiv\left\{\gamma_{5}, \frac{\gamma_{\mu}}{4}, \frac{\gamma_{\mu} \gamma_{5}}{4}, 1\right\} .
$$

For convenience we define:

$$
\begin{gathered}
\Lambda_{q}\left(p^{2}\right)=\frac{-i}{4 N_{c}} \operatorname{tr}\left[\frac{\gamma_{\mu} \sin \left(a p_{\mu}\right)}{\sin ^{2}\left(a p_{\mu}\right)} S^{-1}(p)\right], \\
\Lambda_{\Gamma}\left(p^{2}\right)=-i \frac{\operatorname{tr}\left[\frac{\gamma_{\mu} \sin \left(a p_{\mu}\right)}{\sin ^{2}\left(a p_{\mu}\right)} S^{-1}(p)\right]}{\operatorname{tr}\left[P_{\Gamma} \Pi_{\Gamma}(p)\right]}, \\
\Lambda_{P / S}\left(\mu^{2}\right)=\Lambda_{P}\left(p^{2}\right) / \Lambda_{S}\left(p^{2}\right),
\end{gathered}
$$

such that in the chiral limit

$$
\begin{aligned}
\Lambda_{q}\left(\mu^{2}\right) & =Z_{q}\left(a, \mu^{2}\right), \\
\Lambda_{\Gamma}\left(\mu^{2}\right) & =Z_{\Gamma}\left(a, \mu^{2}\right) \quad \text { and } \\
\Lambda_{P / S}\left(\mu^{2}\right) & =Z_{P}\left(a, \mu^{2}\right) / Z_{S}\left(a, \mu^{2}\right) .
\end{aligned}
$$




\section{B. Evaluation of the correlators}

Following the approach introduced in Ref. [59], we use momentum sources. This approach has the advantage of being computationally inexpensive and it has a high statistical accuracy. We will shortly summarize the procedure.

The vertex functions defined in Eq. (17) are not gauge invariant, and must be computed in a fixed gauge. We chose the Landau gauge by minimizing a functional proposed in Ref. [60].

We introduce $S(y, p)$ which is defined to be the solution of the following linear equation:

$$
\sum_{y} D(x, y) S(y, p)=\mathbb{1} e^{i p x},
$$

where $\mathbb{1}$ stands for the identity matrix in spinor and color indices. It is straightforward to obtain that

$$
G_{\Gamma}(p)=\frac{1}{V} \sum_{z} \gamma_{5} S^{\prime}(z, p)^{\dagger} \gamma_{5} S^{\prime}(z, p),
$$

where $S^{\prime}(z, p)=e^{-i p z} S(z, p)$

and

$$
S(p)=\frac{1}{V} \sum_{x} e^{-i p x} S(x, p) .
$$

\section{Twisted boundary conditions}

In order to interpolate easily between the lattice momenta we use twisted boundary conditions $[61,62]$ by imposing

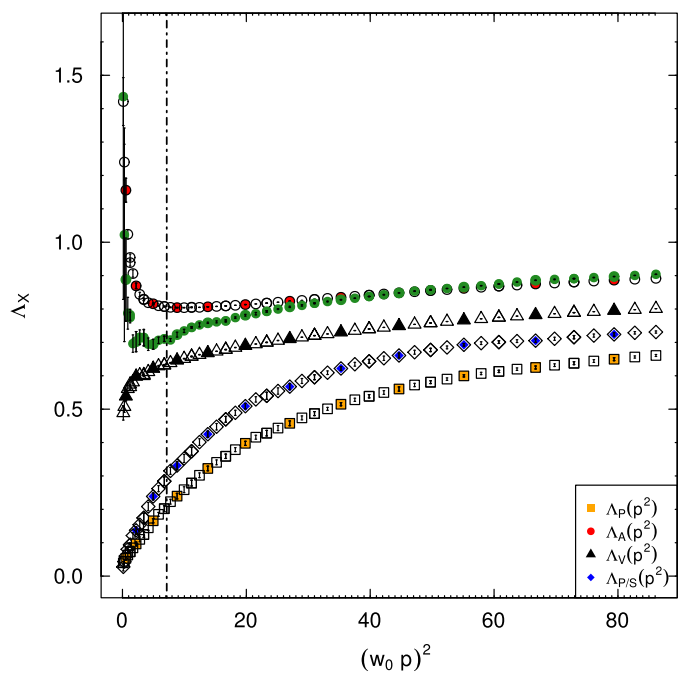

$$
q(x+L)=e^{i B x} q(x) \quad \text { with } \quad B_{\mu}=\frac{\pi \theta_{\mu}}{L_{\mu}},
$$

where $L_{\mu=1,2,3}=L$ and $L_{4}=T$ and $\theta$ is the twist angle. The boundary conditions are imposed by only modifying the Dirac operator in the valence. The accessible momenta are then $p_{\mu}=\frac{2 \pi}{L_{\mu}} n_{\mu}+\frac{\pi}{L_{\mu}} \theta_{\mu}$. Equations (25) and (26) of Sec. IV B can be generalized in the case of twisted boundary conditions.

In practice the propagator $S(p)$ and the Green's function $G_{\Gamma}(p)$ are evaluated for

$$
n_{\mu}=l(1,1,0,0) \quad \text { and } \quad \theta_{\mu}=l^{\prime} \frac{1}{2}(1,1,0,0),
$$

for every pair $\left(l, l^{\prime}\right)$ with $l \in\left[\left[1, \ldots, l_{\max }\right]\right]$ and $l^{\prime} \in\left[\left[-l_{\max }^{\prime}, \ldots, l_{\max }^{\prime}\right]\right]$. Note that we also use negative values for $l^{\prime}$ in order to obtain the same values of $p^{2}$ from twisting with different initial momenta. This is useful in order to estimate cutoff effects. From Fig. 2 it is clear that they are small. Finally, note that we choose "nondemocratic" momenta in Eq. (28).

\section{Results and analysis}

The vertex functions $\Lambda_{X}$ for $X \in\{P, V, A, P / S\}$ at a fixed quark mass, as a function of momentum $(a p)^{2}$, are shown for $\beta=2.0$ and $\beta=2.2$ in Fig. 2. The filled symbols are obtained with twist angle $\theta=0$, while the empty symbol denotes the results obtained for $\theta \neq 0$.

In order to determine the value of the renormalization constants, the first step is to extrapolate the result in the chiral limit. At fixed $p^{2}$ the behavior of the vertex functions, which do not involve the pseudoscalar density,

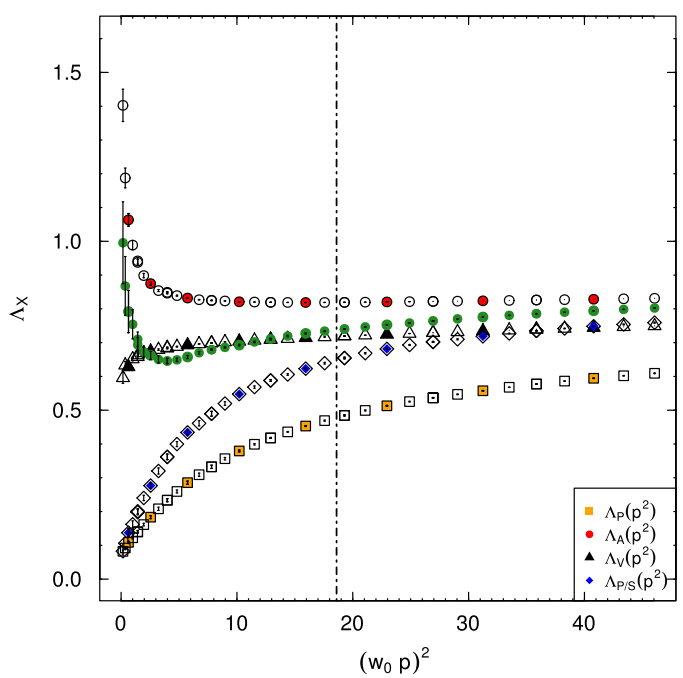

FIG. 2. $\Lambda_{X=P, V, A, P / S}$ as a function of $(a p)^{2}$ for the most chiral point at $\beta=2.0$ (left panel) and $\beta=2.2$ (right panel). The filled data points are obtained without twisted boundary conditions while the empty symbols denote the use of a nonvanishing $\theta$. The vertical lines indicate where $(a p)^{2}=1$. 

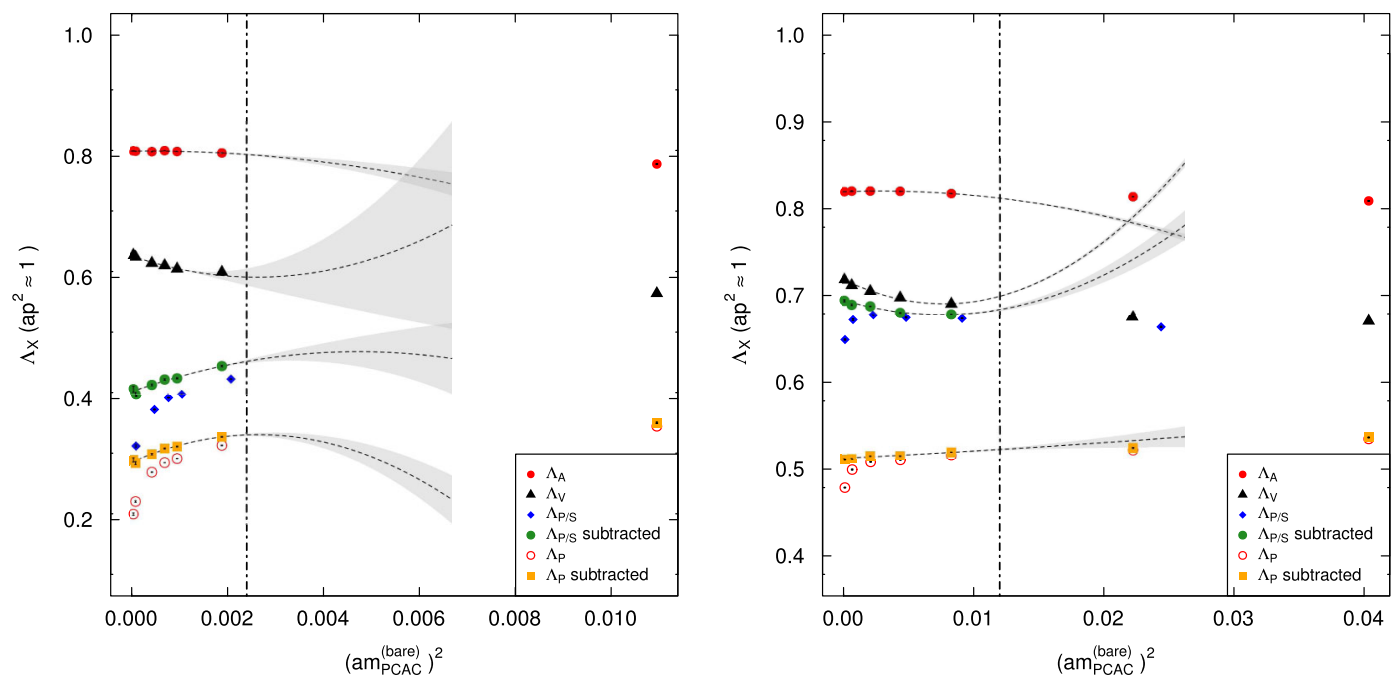

FIG. 3. $\Lambda_{X=P, V, A, P / S}$ at fixed $(a p)^{2}=1$ as a function of $\left(a m_{\mathrm{PCAC}}^{\text {bare }}\right)^{2}$ for $\beta=2.0$ (left panel) and $\beta=2.2$ (right panel).

is expected to be polynomial in $\left(a m_{\mathrm{PCAC}}^{\text {bare }}\right)^{2}$. Concerning the pseudoscalar vertex functions, it is well known that special care must be taken due to the presence of the Goldstoneboson pole $[58,63,64]$. In that case, we use the following ansatz to perform pion-pole subtraction:

$$
\Lambda_{P}\left(p^{2}\right)=\mathcal{A}\left(p^{2}\right)+\mathcal{B}\left(p^{2}\right) m_{\mathrm{PCAC}}+\frac{\mathcal{C}\left(p^{2}\right)}{m_{\mathrm{PCAC}}}
$$

where $\mathcal{A}, \mathcal{B}$ and $\mathcal{C}$ are functions of $p^{2}$. The subtraction is performed for each $p^{2}$ by fitting the data at a given $\beta$, and we will denote by $\Lambda_{P}^{\mathrm{sub}}\left(p^{2}\right)=\Lambda_{P}\left(p^{2}\right)-\frac{\mathcal{C}\left(p^{2}\right)}{m_{\mathrm{PCAC}}}$ the subtracted vertex function at a given fermion mass.

We illustrate the chiral extrapolation at fixed $p^{2}$ in Fig. 3, where we show $\Lambda_{X}\left(p^{2}=1 / a^{2}\right)$ as a function of $\left(a m_{\mathrm{PCAC}}^{\text {bare }}\right)^{2}$. In the plot we also include the Goldstone boson subtracted vertex function for $X=P$ and $P / S$. The chiral extrapolation is obtained by fitting a second-order polynomial in $\left(a m_{\text {PCAC }}^{\text {bare }}\right)^{2}$ to the data. The vertical dasheddotted line indicates the extent of the region included in the fit. The best-fit curve and its statistical error are included in the figure. The typical $\chi^{2} /$ ndof for these fits are much larger than one, due to the small statistical error bars on $\Lambda_{X}$. Therefore, because our data are not well described by our fitting function, we estimate the systematic error by comparing with a first-order extrapolation. This estimate of the systematic error due to the choice of fitting function is reported in Table III. As it can be seen, usually the systematic error is dominant over the statistical one. In the following, we use the combination of systematic and statistical errors for the renormalization constants.

We show in Fig. 4 the dependence of the chirally extrapolated vertex function $\Lambda_{X}$ as a function of $\left(w_{0} p\right)^{2}$. In the continuum, $Z_{V}, Z_{A}$ and $Z_{P} / Z_{S}$ are renormalization scale independent: the observed scale dependence is a manifestation of discretization effects. ${ }^{1}$

In order to have meaningful estimates of $Z_{X}\left(p^{2}\right)$, one relies on the existence of a renormalization window: $\Lambda<p<\mathcal{O}\left(a^{-1}\right)$. The lower bound guarantees that the Goldstone pole contamination is small and that the Wilson coefficient entering in the operator product expansion, which relates the physical process and the matrix element, can be computed in perturbation theory. The upper bound guarantees small lattice artifacts. In our case, reformulating the inequality in units of $w_{0}$, and setting $w_{0}^{\chi} \Lambda \sim w_{0}^{\chi} m_{V} \sim 1$ we have

$$
\left(w_{0}^{\chi} m_{V}\right)^{2} \sim 1<\left(w_{0}^{\chi} p\right)^{2}<\mathcal{O}\left(\left(w_{0}^{\chi} / a\right)^{2}\right) .
$$

Since the smallest value $w_{0}^{\chi} / a$ obtained at $\beta=1.8$ is $w_{0}^{\chi} / a \sim 2$, we would have $1<\left(w_{0}^{\chi} p\right)^{2}<\mathcal{O}(4)$. This is the famous window problem occurring at coarser lattice spacing. We thus have to relax the upper bound of the inequality and introduce larger cutoff effects for our coarser lattices. In practice we chose $\left(w_{0}^{\chi} p\right)^{2}=7$, which corresponds to the lattice cutoff at $\beta=2.0$.

In the following we will check that this particular choice of the reference scale does not affect scale-independent quantities, by using a second reference momentum, at the higher end of the sensible momenta range, namely $\left(w_{0}^{\chi} p\right)^{2}=17$. As shown below, our final results are very stable and do not depend, within errors, on the particular choice of reference momentum.

We summarize the values of the renormalization constants, defined at our reference scale $\left(w_{0}^{\chi} p\right)^{2}=7$, for the four $\beta$ values, in Table III.

\footnotetext{
${ }^{1}$ Note that we do not subtract perturbative $\mathcal{O}(a)$ effects, and we do not convert $Z_{P}\left(\mu^{2}\right)$ to the $\overline{\mathrm{MS}}$ scheme.
} 
TABLE III. Renormalization constant obtained using $\left(w_{0} p\right)^{2}=7$ as a reference scale.

\begin{tabular}{|c|c|c|c|c|c|c|c|c|}
\hline$\beta$ & $Z_{A}$ & $\chi^{2} / \operatorname{dof}$ & $Z_{V}$ & $\chi^{2} /$ dof & $Z_{P} / Z_{S}$ & $\chi^{2} / \operatorname{dof}$ & $Z_{P}^{\mathrm{RI}^{\prime}}$ & $\chi^{2} / \mathrm{dof}$ \\
\hline 1.8 & $0.7791(4)(9)$ & $40 / 1$ & $0.5599(4)(40)$ & $5.6 / 1$ & $0.2809(48)(45)$ & $10.3 / 1$ & $0.2051(36)(66)$ & $5.8 / 1$ \\
\hline 2.0 & $0.8072(3)(5)$ & $44 / 3$ & $0.6356(2)(26)$ & $280 / 3$ & $0.4080(25)(27)$ & $15 / 3$ & $0.2907(16)(72)$ & $15 / 3$ \\
\hline 2.2 & $0.8267(2)(23)$ & $2.9 / 2$ & $0.6973(2)(30)$ & $107 / 2$ & $0.5655(16)(121)$ & $31 / 2$ & $0.3803(8)(49)$ & $19 / 2$ \\
\hline 2.3 & $0.8449(23)(72)$ & $0 / 0$ & $0.7280(19)(80)$ & $0 / 0$ & $0.6799(260)(440)$ & $0 / 0$ & $0.4201(136)(13)$ & $0 / 0$ \\
\hline
\end{tabular}

\section{SPECTROSCOPY}

\section{A. Effective masses}

We compute the mass of the lightest (isovector) pseudoscalar, vector and axial-vector meson resonances using two-point correlators. As explained in Sec. II, the mass can be extracted using the large-time behavior of the effective mass as described by Eq. (4). This approach is justified if the state is stable. We illustrate effective masses for various ensembles in Figs. 5,6,7 and 8.

The effective masses are fitted on a given plateau range, which is determined for each state by individual inspection. Systematic errors introduced by the choice of the plateaux are small for the pseudoscalar and vector resonances, and for this reason we will neglect them in the following. The best-fit value for the effective mass is plotted for each state in the figures together with its statistical error. The masses of the vector and pseudoscalar mesons are clearly determined for all ensembles. For the axial-vector correlator we do not observe long plateaux, due to the much worse signal-to-noise ratio as a function of Euclidean time separation. This results in significantly larger systematic errors, which are not yet fully under control.

In each plot, we also show the two- and three-pion thresholds. This shows that the vector-meson resonance, whose main decay channel is expected to be the decay in two pions, is stable for almost all of our simulations. In a

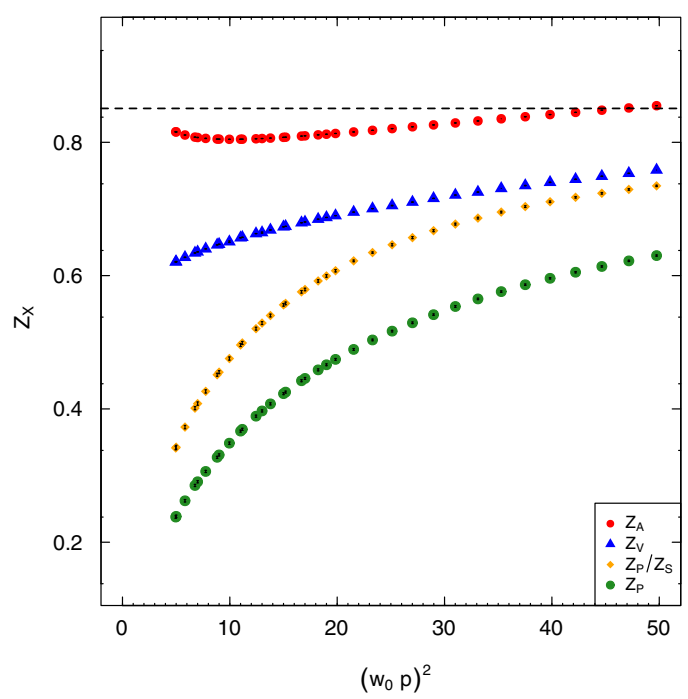

few cases, our most chiral points at $\beta=1.8$ and $\beta=2.0$ are at kinematical threshold. A similar conclusion can be drawn for the isovector axial-vector meson, whose main decay channel is expected to be three pions.

\section{B. $\boldsymbol{m}_{\mathrm{PS}}$ and $\boldsymbol{F}_{\mathrm{PS}}$}

The continuum expressions for $m_{\mathrm{PS}}$ and $F_{\mathrm{PS}}$ have been worked out in Ref. [65] at next-to-leading order in chiral perturbation theory:

$$
\begin{aligned}
\frac{m_{\mathrm{PS}}^{2}}{m_{\mathrm{f}}} & =2 B\left[1+\frac{3}{4} x \log \frac{2 B m_{\mathrm{f}}}{\mu^{2}}+b_{M} x+\mathcal{O}\left(x^{2}\right)\right], \\
F_{\mathrm{PS}} & =F\left[1-x \log \frac{2 B m_{\mathrm{f}}}{\mu^{2}}+b_{F} x+\mathcal{O}\left(x^{2}\right)\right],
\end{aligned}
$$

where $x=\frac{2 B m_{\mathrm{f}}}{(4 \pi F)^{2}}$ and $m_{\mathrm{f}}$ is the renormalized fermion mass at a given scale. In the conventions of Ref. [65], the condensate is given by $\Sigma \equiv-2 B F^{2}$. Note that $F$ and $B$ appear in both expressions. Note that we are using the chiral perturbation theory formulas for infinite volume, which, given the values of $F_{\mathrm{PS}} L$ reported in Appendix A, appear to be valid for our data. The mass range of applicability of the effective theory is not known a priori. In order to make the fits more stable, we will rewrite the

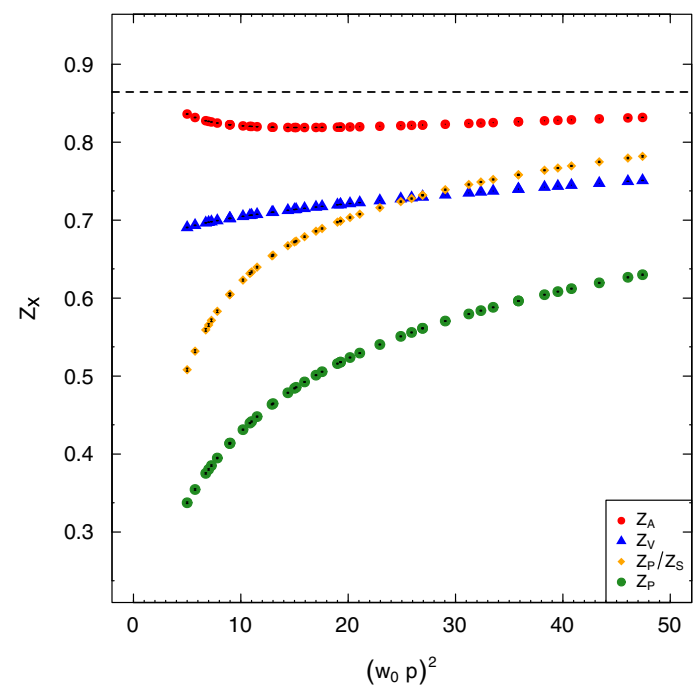

FIG. 4. $Z_{X=P, V, A, P / S}$ as function of the renormalization scale $\left(w_{0} \mu\right)^{2}=\left(w_{0} p\right)^{2}$ for $\beta=2.0$ (left panel) and $\beta=2.2$ (right panel). 


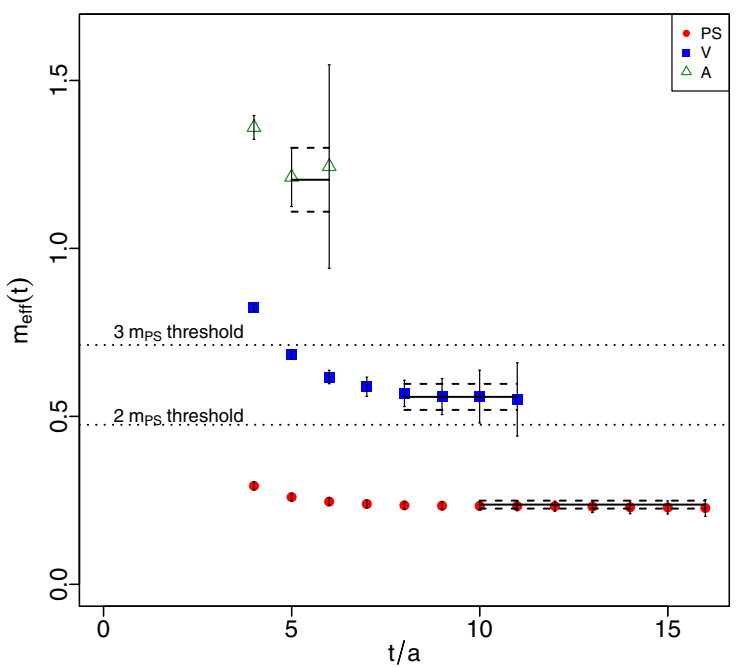

FIG. 5. Effective masses of the pseudoscalar, vector and axial meson masses $\left(\beta=1.8, m_{0}=-1.157, L=24\right)$.

expansion in a new parameter, $\tilde{x}=\frac{m_{\mathrm{PS}}^{2}}{(4 \pi F)^{2}}$. At this order Eq. (31) and Eq. (32) remain unchanged (this is, however, not true at NNLO) and read

$$
\begin{aligned}
& \frac{m_{\mathrm{PS}}^{2}}{m_{\mathrm{f}}}=2 B\left[1+\frac{3}{4} \tilde{x} \log \frac{m_{\mathrm{PS}}^{2}}{\mu^{2}}+b_{M} \tilde{x}+\mathcal{O}\left(\tilde{x}^{2}\right)\right], \\
& F_{\mathrm{PS}}=F\left[1-\tilde{x} \log \frac{m_{\mathrm{PS}}^{2}}{\mu^{2}}+b_{F} \tilde{x}+\mathcal{O}\left(\tilde{x}^{2}\right)\right] .
\end{aligned}
$$

From this result we observe that the expansion of $F_{\mathrm{PS}}$ now is independent of $B$, which will allow us to perform the fit in two steps: first a fit to $F_{\mathrm{PS}}$ to obtain $F$ and then using it as an input for a second fit to $m_{\mathrm{PS}}^{2} / m_{\mathrm{f}}$ to obtain $B$.

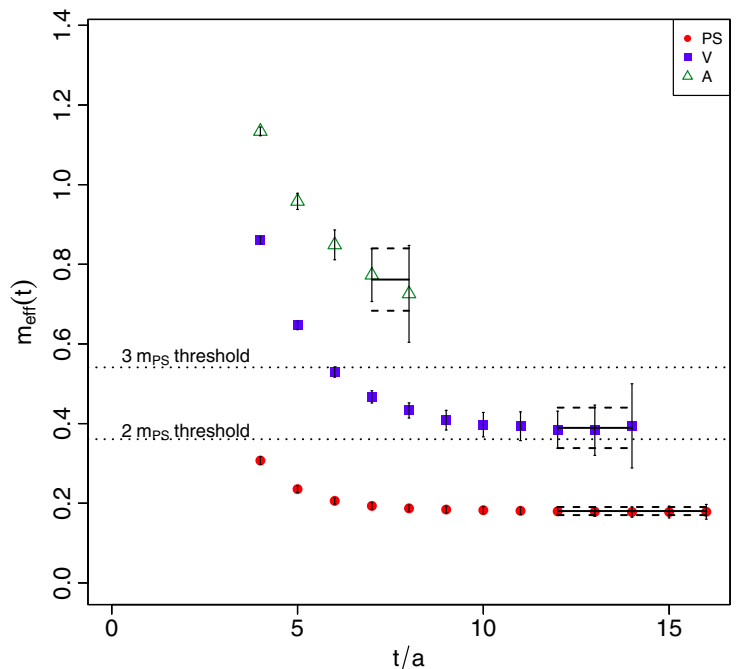

FIG. 6. Effective masses of the pseudoscalar, vector and axial meson masses $\left(\beta=2.0, m_{0}=-0.958, L=32\right)$.

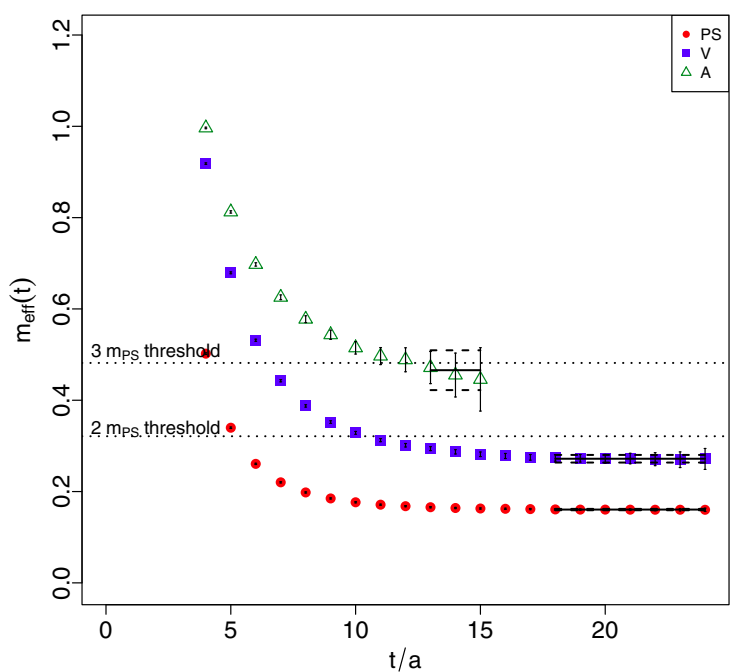

FIG. 7. Effective masses of the pseudoscalar, vector and axial meson masses $\left(\beta=2.2, m_{0}=-0.76, L=48\right)$.

The renormalized values for $F_{\mathrm{PS}}$ and $m_{\mathrm{PS}}^{2} / m_{\mathrm{f}}$ at four values of the lattice spacing are shown as a function of $m_{\mathrm{PS}}^{2}$ in Figs. 9 and 10. All the lattices included in the fit satisfy $m_{\mathrm{PS}} L \geq 5$.6. The fermion mass is given by $m_{\mathrm{f}}\left(p^{2}\right)=$ $m_{\mathrm{PCAC}} Z_{A} / Z_{P}\left(p^{2}\right)$ and the renormalized pseudoscalar decay constant is $F_{\mathrm{PS}}=F_{\mathrm{PS}}^{(\text {bare })} Z_{A}$. As a reference scale for the renormalization constants we use $p=\sqrt{7} / w_{0}^{\chi}$. As can be seen, significant cutoff effects are observed. In order to estimate the low-energy constants $F$ and $B$ in the continuum, discretization effects must then be taken into account. In order to obtain a reliable estimate, we will use two different strategies.

The first strategy (strategy I) is based on fitting the pseudoscalar mass and decay constant using several lattice spacings simultaneously together with a given model for the lattice discretization effects:

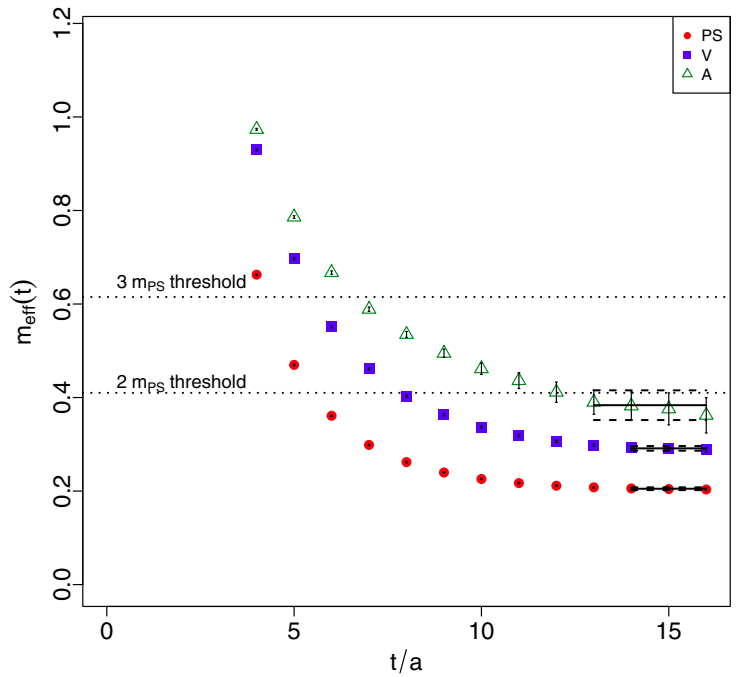

FIG. 8. Effective masses of the pseudoscalar, vector and axial meson masses $\left(\beta=2.3, m_{0}=-0.675, L=32\right.$ ). 


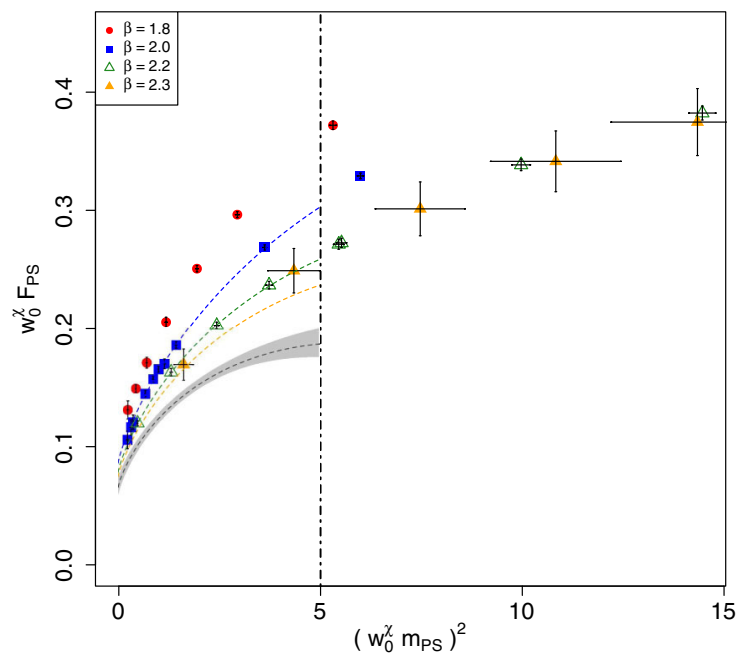

FIG. 9. $\quad F_{\mathrm{PS}}$ versus $m_{\mathrm{PS}}^{2}$ for the four lattice spacings. The curves correspond to the best-fit parameters obtained fitting only $\beta=2.0, \beta=2.2$ and $\beta=2.3$ (subset $S_{2}$ ) and are drawn for the corresponding lattice spacing. The black curve indicates the continuum results.

$$
\frac{m_{\mathrm{PS}}^{2}}{m_{\mathrm{f}}}=2 B\left[1-a_{M} \tilde{x} \log \frac{m_{\mathrm{PS}}^{2}}{\mu^{2}}+b_{M} \tilde{x}+\delta_{M} \frac{a}{w_{0}^{\chi}}+\gamma_{M} m_{\mathrm{PS}}^{2} \frac{a}{w_{0}^{\chi}}\right],
$$

$$
F_{\mathrm{PS}}=F\left[1-a_{F} \tilde{x} \log \frac{m_{\mathrm{PS}}^{2}}{\mu^{2}}+b_{F} \tilde{x}+\delta_{F} \frac{a}{w_{0}^{\chi}}+\gamma_{F} m_{\mathrm{PS}}^{2} \frac{a}{w_{0}^{\chi}}\right] .
$$

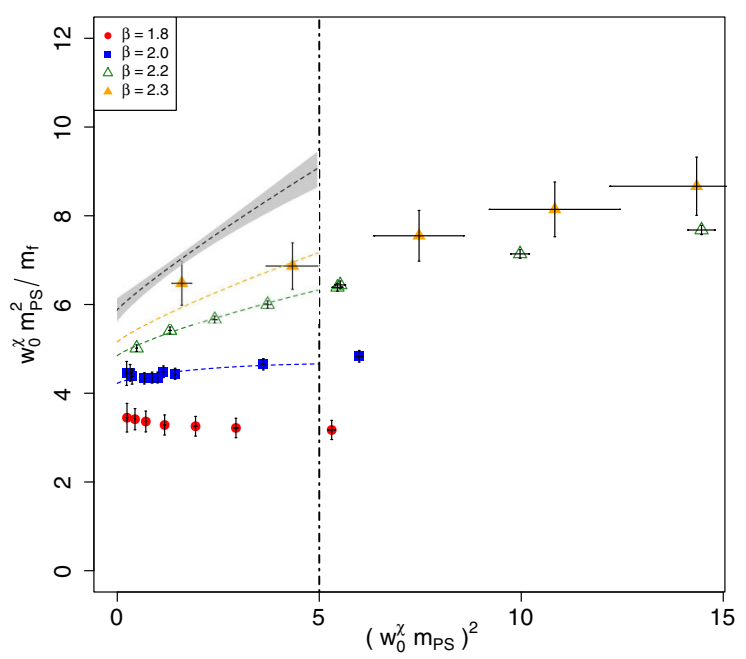

FIG. 10. $m_{\mathrm{PS}}^{2} / m_{\mathrm{f}}$ versus $m_{\mathrm{PS}}^{2}$ for the four lattice spacings. The curves correspond to the best-fit parameters obtained fitting only $\beta=2.0, \beta=2.2$ and $\beta=2.3$ (subset $S_{2}$ ) and are drawn for the corresponding lattice spacing. The black curve indicates the continuum results.
Here the new fitting parameters $\delta_{M, F}$ and $\gamma_{M, F}$ control the discretization effects. Note that the two coefficients $a_{F, M}$ are fixed in the continuum, but here we consider them as free parameters.

To control the stability of the fit, we consider four subsets of our data $S_{1}=\{\beta=2.0,2.2\}, S_{2}=\{\beta=2.0,2.2,2.3\}$, $S_{3}=\{\beta=1.8,2.0,2.2\}$ and $S_{4}=\{\beta=1.8,2.0,2.2,2.3\}$ and perform the fit on each of these subsets. The result of the fit for the $S_{2}$ subset is shown in Figs. 9 and 10.

The second strategy (strategy II) consists of fitting each of the lattice spacings independently, to obtain the coefficients $B, F, a_{F, M}$ and $b_{F, M}$, while setting to zero the coefficients $\delta_{M, F}, \gamma_{M, F}$ in Eqs. (35) and (36). In a second step, lattice discretization effects can be assessed by studying the dependence of the coefficients as a function of the lattice spacing.

In all fits we use $w_{0}^{\chi} \mu=1$ as a scale. The results of the fits, including their $\chi^{2}$ per degrees of freedom, are summarized in Table IV for strategy I and Table V for strategy II. The fits are performed on a given range of values for $\left(w_{0}^{\chi} m_{\mathrm{PS}}\right)^{2}$ below the "cut" given in the tables.

Strategy II allows us to extract an estimate of $w_{0}^{\chi} F$ and $w_{0}^{\chi} B$ for each lattice spacing. This is shown in Fig. 11, where the value of $B$ has been rescaled by a factor of 20 for convenience. The scaling towards the continuum limit is compatible with a linear behavior and no $\mathcal{O}\left(a^{2}\right)$ effects are visible. On the plot we also show the results obtained directly in the continuum using the first strategy for the subset of gauge ensembles $S_{1}$ and $S_{2}$. The results obtained with strategy I for the subsets $S_{3}$ and $S_{4}$ have a $\chi^{2} /$ ndof $\sim 10$ and thus do not describe the data well.

Our final estimates for the chiral parameters are $w_{0}^{\chi} B=$ 2.88(15)(17) and $w_{0}^{\chi} F=0.078(4)(12)$. The central value and statistical error comes from the linear extrapolation to the continuum of the fits at fixed beta (strategy I). The systematic error is obtained by computing the maximal difference between the results obtained by strategy I and II. By setting the scale to $F=246 \mathrm{GeV}$ one arrives at the result $w_{0}^{\chi}=6.3(3)(9) \times 10^{-5} \mathrm{fm}$. The value of the condensate then reads $\Sigma^{1 / 3} / F=4.19(26)$ (statistical and systematical errors have been combined).

We repeated a similar analysis using $p=\sqrt{17} / w_{0}^{\chi}$ as a reference scale, which is shown in Appendix B. As claimed in the previous section, we do not observe any statistically significant change in the continuum values of $F$ and $B$.

\section{Heavier states}

In this section we report our results for the mass of two heavier isotriplet meson resonances, namely the vector in Fig. 12 and the axial-vector in Fig. 13. All the masses are presented in units of $w_{0}^{\chi}$ as functions of $\left(w_{0}^{\chi} m_{\mathrm{PS}}\right)^{2}$. In each figure we present a global fit, including all the available data at four lattice spacings, to the following fit ansatz: 
TABLE IV. Results of the global fits of $m_{\mathrm{PS}}^{2} / m_{\mathrm{f}}$ and $F_{\mathrm{PS}}$ on subset $S_{1,2,3,4}$ using $\left(w_{0} p\right)^{2}=7$ as a reference renormalization scale.

\begin{tabular}{|c|c|c|c|c|c|}
\hline Type & Coefficient & $S_{1}$ & $S_{2}$ & $S_{3}$ & $S_{4}$ \\
\hline NLO global & $\mathrm{F}$ & $0.066(6)$ & $0.066(6)$ & $0.049(5)$ & $0.049(5)$ \\
\hline NLO global & $b_{F}$ & $0.0038(2)$ & $0.0038(2)$ & $0.0028(1)$ & $0.0028(1)$ \\
\hline NLO global & $\delta_{F}$ & $0.05(1)$ & $0.05(1)$ & $0.09(1)$ & $0.09(1)$ \\
\hline NLO global & $\gamma_{F}$ & $0.05(1)$ & $0.051(9)$ & $0.072(6)$ & $0.069(6)$ \\
\hline NLO global & $a_{F}$ & $0.22(3)$ & $0.21(2)$ & $0.19(1)$ & $0.18(1)$ \\
\hline NLO global & $\chi^{2} /$ ndof & $9.7 / 8$ & $13 . / 10$ & $83 . / 14$ & $91 . / 16$ \\
\hline NLO global & cut & 5 & 5 & 5 & 5 \\
\hline NLO global & B & $2.9(1)$ & $2.9(1)$ & $3.0(1)$ & $3.0(1)$ \\
\hline NLO global & $b_{M}$ & $0.0005(1)$ & $0.0005(1)$ & $0.00028(8)$ & $0.00029(8)$ \\
\hline NLO global & $\delta_{M}$ & $-0.7(1)$ & $-0.74(9)$ & $-0.84(7)$ & $-0.85(6)$ \\
\hline NLO global & $\gamma_{M}$ & $-0.25(7)$ & $-0.25(7)$ & $-0.24(5)$ & $-0.24(5)$ \\
\hline NLO global & $a_{M}$ & $0.00(1)$ & $0.00(1)$ & $0.003(5)$ & $0.003(5)$ \\
\hline NLO global & $\chi^{2} /$ ndof & $10 . / 8$ & 14./10 & $27 . / 14$ & $30 . / 16$ \\
\hline NLO global & cut & 5 & 5 & 5 & 5 \\
\hline
\end{tabular}

TABLE V. Results of the fixed lattice spacing fits for each $\beta$ value using $\left(w_{0} p\right)^{2}=7$ as a reference renormalization scale.

\begin{tabular}{lccccc}
\hline \hline Type & Coefficient & $\beta=1.8$ & $\beta=2.0$ & $\beta=2.2$ & $\beta=2.3$ \\
\hline NLO fixed $\beta$ & $\mathrm{F}$ & $0.096(4)$ & $0.088(3)$ & $0.086(3)$ & $0.09(4)$ \\
NLO fixed $\beta$ & $a_{F}$ & $0.41(2)$ & $0.27(1)$ & $0.1(1)$ \\
NLO fixed $\beta$ & $b_{F}$ & $0.0093(1)$ & $0.0066(1)$ & $0.0052(1)$ & $6.8 / 4$ \\
NLO fixed $\beta$ & $\chi^{2} /$ ndof & $4.1 / 4$ & $9.3 / 7$ & 12 & $1.1 / 1$ \\
NLO fixed $\beta$ & cut & 12 & $2.18(8)$ & 12 \\
NLO fixed $\beta$ & $\mathrm{B}$ & $1.7(1)$ & $-0.00(2)$ & $0.025(7)$ & $0.01(7)$ \\
NLO fixed $\beta$ & $a_{M}$ & $-0.02(6)$ & $0.0000(3)$ & $0.0007(1)$ & $5.0 / 4$ \\
NLO fixed $\beta$ & $b_{M}$ & $-0.0004(7)$ & $8.2 / 7$ & 12 & $0.000(1)$ \\
NLO fixed $\beta$ & $\chi^{2} /$ ndof & $4.0 / 4$ & 12 & $1.0 / 1$ \\
NLO fixed $\beta$ & cut & 12 & & 12 \\
\hline \hline
\end{tabular}

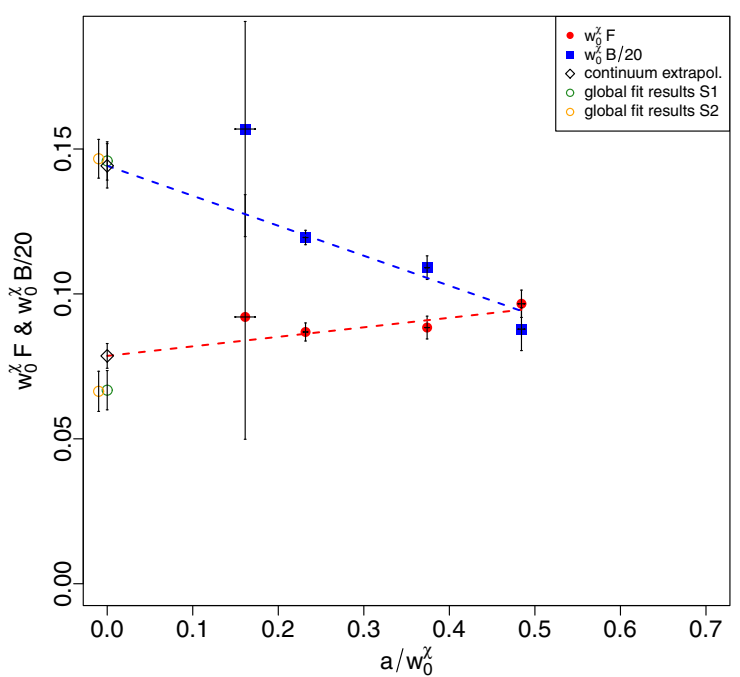

FIG. 11. Values of the chiral parameters $B$ and $F$ in units of the reference lattice scale $w_{0}^{\chi}$ as extracted using strategy II described in the text. In this plot $B$ has been rescaled by a factor of 20 for graphical convenience.

$$
w_{0}^{\chi} m_{X}=w_{0}^{\chi} m_{X}^{\chi}+A\left(w_{0}^{\chi} m_{\mathrm{PS}}\right)^{2}+B\left(w_{0}^{\chi} m_{\mathrm{PS}}\right)^{4}+C \frac{a}{w_{0}} .
$$

The fit range for each channel is shown by the vertical dotted line in the plot. The gray band indicates the $1 \sigma$ error band for the continuum prediction, obtained by setting $a=0$ with our best-fit parameters. The results of the fit for the axial and vector meson are summarized in Table VI.

TABLE VI. Results of the polynomial fits of the vector and axial resonances.

\begin{tabular}{lcc}
\hline \hline Coefficient & Vector & Axial \\
\hline$w_{0}^{\chi} m_{X}$ & $1.01(3)$ & $1.1(1)$ \\
$A$ & $0.47(3)$ & $0.8(1)$ \\
$B$ & $-0.039(6)$ & $-0.09(3)$ \\
$C$ & $-0.05(7)$ & $2.1(3)$ \\
$\chi^{2} /$ ndof & $23 / 16$ & $20 / 16$ \\
Cut & 4 & 4 \\
\hline \hline
\end{tabular}




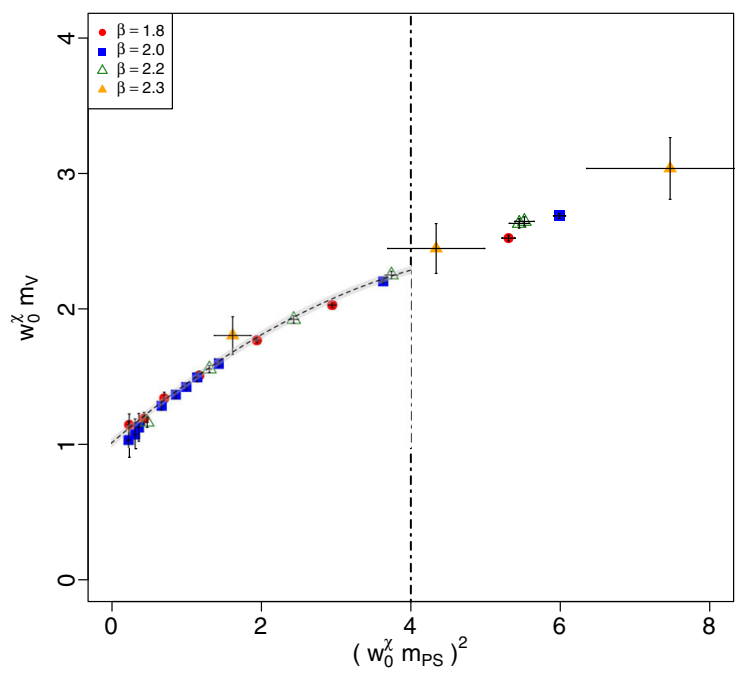

FIG. 12. Combined chiral and continuum extrapolation of the vector-meson mass $m_{V}$. Our data for four lattice spacings is presented together with the best fit at each lattice spacing. The grey band is our result for the continuum extrapolation and its $1 \sigma$ confidence region.

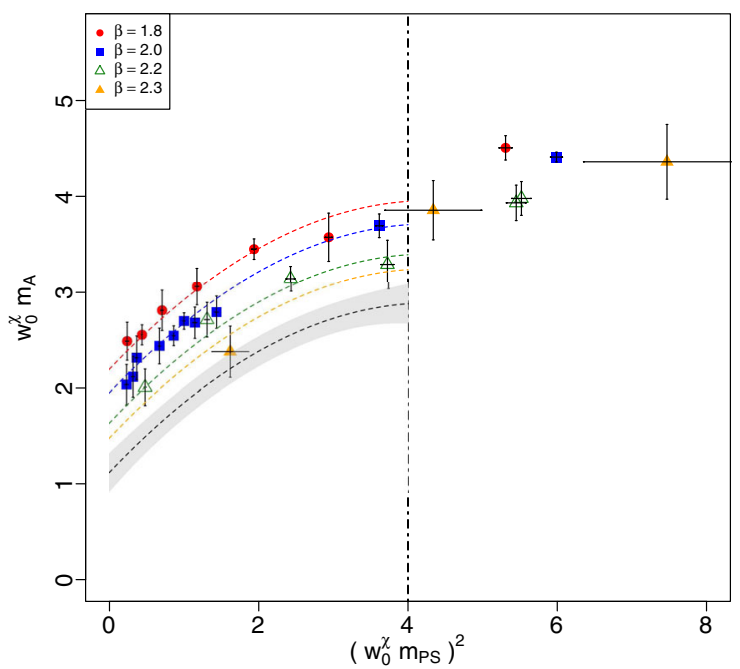

FIG. 13. Combined chiral and continuum extrapolation of the axial-vector meson mass $m_{A}$. Our data for four lattice spacings is presented together with the best fit at each lattice spacing. The grey band is our result for the continuum extrapolation and its $1 \sigma$ confidence region.

For the vector meson the fit describes our data well and the observed cutoff effects are small. We find $w_{0}^{\chi} m_{V}^{\chi}=1.01(3)$ with a $\chi^{2} /$ ndof $=23 / 16$. Note that for our data $m_{V}$ is always less than $2 m_{\mathrm{PS}}$, except maybe for the most chiral point used in the fit, so that the vector meson is expected to be stable and its mass can be reliably extracted from the large (Euclidean) time behavior of the appropriate two-point function.

For the mass of the axial-vector meson, our data is more noisy already at the level of the effective masses and we therefore have larger systematic uncertainties. The ansatz (37) fits the data well, within large errors, and the resulting value for the mass is $w_{0}^{\chi} m_{A}^{\chi}=1.1(1)$ with $\chi^{2} /$ ndof $=$ 20/16. In units of $F_{\mathrm{PS}}$ we have $m_{V} / F_{\mathrm{PS}} \sim 13.1(2.2)$ and $m_{A} / F_{\mathrm{PS}} \sim 14.5(3.6)$.

\section{CONCLUSION}

We analyzed the $\mathrm{SU}(2)$ gauge theory with $N_{f}=2$ flavors of fermions in the fundamental representation using lattice techniques. Dynamical simulations have been performed at four lattice spacings and a number of volumes and masses to asses systematic effects and to carry out the necessary extrapolations. We determined nonperturbatively, in the RI'-MOM scheme, the relevant renormalization constants and performed a detailed analysis of the mass and decay constant of the pseudoscalar Goldstone bosons, including an extrapolation to the chiral and continuum limits to take into account the lattice cutoff effects present in our computation. We used a conservative estimate of all systematic uncertainties to obtain a reliable estimate of $F_{\mathrm{PS}}$. Finally we analyzed the mass of the spin- 1 bound states and determined the ratios $m_{V} / F_{\mathrm{PS}}=13.1(2.2)$ and $m_{A} / F_{\mathrm{PS}}=$ 14.5(3.6) for the continuum theory in the chiral limit, using similar extrapolation methods. Our final results are consistent with, and improve upon, previous results for this model, which were performed with only two lattice spacings, at much larger quark masses and using a perturbative estimate of the renormalization constants. In the case of the axial resonance, our result is affected by a systematic error, not included in the above estimate, stemming from higherstates contamination in the extraction of the effective mass resulting in short plateaux for the two coarser lattices used in this work. We estimated the size of this error due to the choice of the plateaux on the final value of $m_{A}$ to be of the same order as the quoted error.

In the context of the fundamental composite (Goldstone) Higgs dynamics [24] our results predict new resonances of mass

$$
m_{V}=\frac{3.2(5)}{\sin \theta} \mathrm{TeV}, \quad \text { and } \quad m_{A}=\frac{3.6(9)}{\sin \theta} \mathrm{TeV},
$$

which are beyond the present LHC constraints, even in the technicolor limit [6] where $\theta=\pi / 2$.

In the context of dark matter models, in particular for the SIMPlest case, because the dark pion is estimated to be around 10 times its decay constant [38], we cannot use the estimate above. Nonetheless, a preliminary result can be obtained from our simulations reported in the first line of Table VII, at $\beta=2$, which yield $m_{\mathrm{PS}} / F_{\mathrm{PS}} \approx 7.5, m_{V} / F_{\mathrm{PS}} \approx$ 8.3 and $m_{A} / F_{\mathrm{PS}} \approx 13.7$. Although these results need crucial refinement they immediately show that for such large values of the dark pion mass one cannot neglect the effects of higher mass states since the overall spectrum is much more compressed than in the case of the chiral limit. 


\section{ACKNOWLEDGMENTS}

This work was supported by the Danish National Research Foundation DNRF:90 grant and by a Lundbeck Foundation Fellowship grant. We acknowledge Partenership for Advanced Computing in Europe (PRACE) for awarding us access to computational resources on MareNostrum at the Barcelona Supercomputing Centre, Spain. Additional local computational facilities used in this work were provided by the local HS9 cluster, funded by a Danish e-infrastructure Cooperation (DeIC) grant, and by the DeIC national High Performance Computing (HPC) centre at University of Southern Denmark (SDU), funded by SDU.

\section{APPENDIX A: NUMERICAL RESULTS}

We report in this section our numerical results for the main spectroscopy quantities studied in this article. The column "stat" reports the number of thermalized configurations used in the analysis, while the column " $N_{\text {rep }}$ " is the number of "replicas" runs used, i.e., the number of independent runs with the same bare parameters.

Some of the masses reported in Table VII show statistically significant differences with respect to the values reported in Ref. [1]. These deviations are, in all cases, below $10 \%$ and in many cases much lower. Such deviations are due to the more limited statistics used in the previous publication which resulted in some residual thermalization effects, and, in some cases, an underestimation of autocorrelation times.

For the reader's convenience, we also report in Table VIII the values of $F_{\mathrm{PS}} L$ for the most chiral point at each lattice spacing.

\section{APPENDIX B: SYSTEMATIC ERROR DUE TO THE CHOICE OF RENORMALIZATION SCALE}

In this appendix we report the dependence of our continuum extrapolation results for the low-energy

TABLE VII. Numerical results for large-volume runs used in the analysis presented in this paper.

\begin{tabular}{|c|c|c|c|c|c|c|c|c|c|c|c|}
\hline$\beta$ & $\mathrm{L}$ & $\mathrm{T}$ & $m_{0}$ & $N_{\text {rep }}$ & Statistics & $m_{\mathrm{PS}} L$ & $m_{\text {PCAC }}^{\text {(bare) }}$ & $m_{\mathrm{PS}}$ & $F_{\mathrm{PS}}^{(\text {bare })}$ & $m_{V}$ & $m_{A}$ \\
\hline 1.8 & 16 & 32 & 1 & 1 & 1562 & 17.85136 & $0.2133(2)$ & $1.115(1)$ & $0.231(1)$ & $1.221(1)$ & $2.20(3)$ \\
\hline 1.8 & 16 & 32 & 1.089 & 2 & 19986 & 13.27994 & $0.11638(7)$ & $0.8299(3)$ & $0.1842(3)$ & $0.9831(9)$ & $1.7(1)$ \\
\hline 1.8 & 16 & 32 & 1.12 & 1 & 3168 & 10.78498 & $0.0758(2)$ & $0.674(1)$ & $0.155(1)$ & $0.857(4)$ & $1.6(2)$ \\
\hline 1.8 & 16 & 32 & 1.14 & 1 & 1225 & 8.386688 & $0.0454(5)$ & $0.524(3)$ & $0.127(2)$ & $0.73(1)$ & $1.5(1)$ \\
\hline 1.8 & 16 & 32 & 1.15 & 1 & 1517 & 6.51176 & $0.0267(5)$ & $0.406(4)$ & $0.106(2)$ & $0.65(2)$ & $1.3(1)$ \\
\hline 1.8 & 24 & 32 & 1.155 & 1 & 3316 & 7.696368 & $0.0163(3)$ & $0.320(3)$ & $0.092(1)$ & $0.58(2)$ & $1.2(2)$ \\
\hline 1.8 & 24 & 32 & 1.157 & 1 & 1447 & 5.70156 & $0.0088(7)$ & $0.23(1)$ & $0.081(4)$ & $0.55(3)$ & $1.34(9)$ \\
\hline 2 & 16 & 32 & 0.85 & 2 & 46057 & 14.64522 & $0.1669(1)$ & $0.9153(5)$ & $0.1524(3)$ & $1.0050(8)$ & $1.64(3)$ \\
\hline 2 & 16 & 32 & 0.9 & 2 & 20316 & 11.37803 & $0.1046(2)$ & $0.711(1)$ & $0.1244(5)$ & $0.824(1)$ & $1.39(5)$ \\
\hline 2 & 16 & 32 & 0.94 & 2 & 9377 & 7.160768 & $0.0434(3)$ & $0.447(2)$ & $0.086(1)$ & $0.598(6)$ & $1.07(7)$ \\
\hline 2 & 16 & 32 & 0.945 & 1 & 3760 & 6.399184 & $0.0343(6)$ & $0.399(4)$ & $0.078(1)$ & $0.56(1)$ & $1.07(7)$ \\
\hline 2 & 32 & 32 & 0.947 & 2 & 1826 & 11.96282 & $0.0309(3)$ & $0.373(2)$ & $0.0765(9)$ & $0.535(7)$ & $1.01(7)$ \\
\hline 2 & 32 & 32 & 0.949 & 4 & 1633 & 11.10832 & $0.0266(3)$ & $0.347(2)$ & $0.072(1)$ & $0.51(1)$ & $0.96(7)$ \\
\hline 2 & 32 & 32 & 0.952 & 1 & 2005 & 9.80432 & $0.0208(3)$ & $0.306(3)$ & $0.067(1)$ & $0.48(1)$ & $0.94(8)$ \\
\hline 2 & 32 & 32 & 0.957 & 1 & 711 & 6.762944 & $0.0096(5)$ & $0.211(6)$ & $0.054(2)$ & $0.40(4)$ & $0.95(5)$ \\
\hline 2 & 32 & 32 & 0.958 & 1 & 957 & 5.772608 & $0.0070(6)$ & $0.18(1)$ & $0.049(3)$ & $0.38(5)$ & $0.84(9)$ \\
\hline 2.2 & 16 & 32 & 0.6 & 1 & 256 & 14.10846 & $0.2008(3)$ & $0.881(1)$ & $0.107(1)$ & $0.925(2)$ & $1.31(2)$ \\
\hline 2.2 & 16 & 32 & 0.65 & 1 & 512 & 11.71451 & $0.1489(3)$ & $0.732(1)$ & $0.0949(7)$ & $0.787(2)$ & $1.17(4)$ \\
\hline 2.2 & 16 & 32 & 0.68 & 1 & 2894 & 8.716016 & $0.0914(2)$ & $0.544(1)$ & $0.0764(6)$ & $0.613(2)$ & $0.92(3)$ \\
\hline 2.2 & 16 & 32 & 0.7 & 1 & 2148 & 8.660944 & $0.0909(3)$ & $0.541(2)$ & $0.0760(7)$ & $0.610(3)$ & $0.91(4)$ \\
\hline 2.2 & 32 & 32 & 0.72 & 2 & 4437 & 14.31942 & $0.0660(4)$ & $0.4474(7)$ & $0.0663(4)$ & $0.521(1)$ & $0.81(1)$ \\
\hline 2.2 & 32 & 32 & 0.735 & 4 & 1257 & 11.55728 & $0.0456(2)$ & $0.361(1)$ & $0.0567(4)$ & $0.446(3)$ & $0.72(3)$ \\
\hline 2.2 & 32 & 32 & 0.75 & 5 & 196 & 8.477056 & $0.0257(4)$ & $0.264(1)$ & $0.0456(6)$ & $0.362(4)$ & $0.62(3)$ \\
\hline 2.2 & 48 & 48 & 0.76 & 1 & 1409 & 7.70736 & $0.0101(1)$ & $0.160(1)$ & $0.0337(4)$ & $0.271(8)$ & $0.45(7)$ \\
\hline 2.3 & 32 & 32 & 0.575 & 2 & 717 & 19.54832 & $0.1327(2)$ & $0.610(1)$ & $0.0715(5)$ & $0.648(2)$ & $0.89(2)$ \\
\hline 2.3 & 32 & 32 & 0.6 & 2 & 4750 & 16.98768 & $0.1066(1)$ & $0.5308(6)$ & $0.0651(1)$ & $0.5731(9)$ & $0.82(1)$ \\
\hline 2.3 & 32 & 32 & 0.625 & 2 & 1233 & 14.10864 & $0.0793(2)$ & $0.440(1)$ & $0.0575(4)$ & $0.489(2)$ & $0.72(1)$ \\
\hline 2.3 & 32 & 32 & 0.65 & 2 & 2296 & 10.75213 & $0.0506(2)$ & $0.336(1)$ & $0.0475(2)$ & $0.394(2)$ & $0.62(1)$ \\
\hline 2.3 & 32 & 32 & 0.675 & 2 & 1436 & 6.560576 & $0.0199(3)$ & $0.205(3)$ & $0.0323(5)$ & $0.291(4)$ & $0.38(5)$ \\
\hline
\end{tabular}

TABLE VIII. Values of $F_{\mathrm{PS}} L$ for the most chiral point at each lattice spacing.

\begin{tabular}{lllll}
\hline \hline$\beta$ & 1.8 & 2.0 & 2.2 & 2.3 \\
$F_{\mathrm{PS}} L$ & 1.49 & 1.25 & 1.29 & 0.86 \\
\hline \hline
\end{tabular}




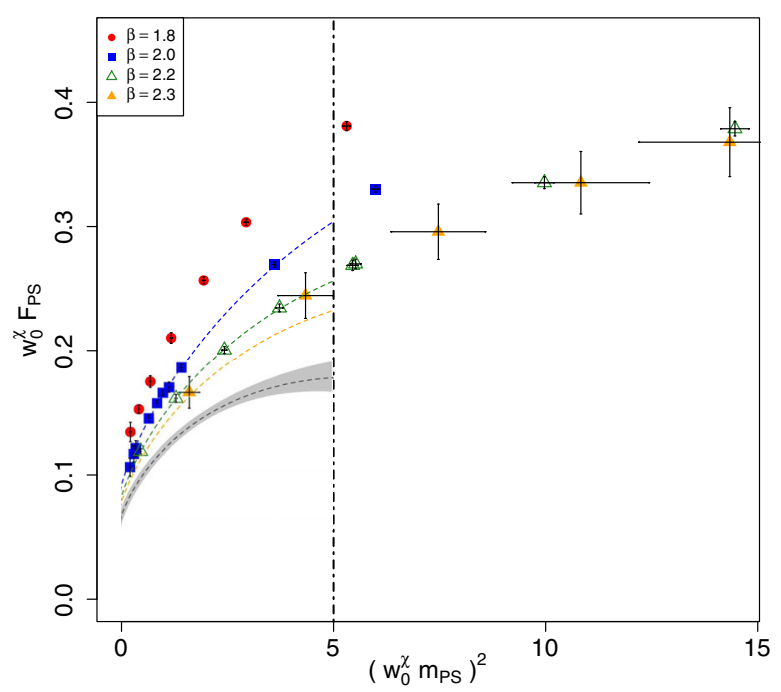

FIG. 14. $F_{\mathrm{PS}}$ versus $m_{\mathrm{PS}}^{2}$ for four lattice spacings, using $\left(w_{0}^{\chi} p\right)^{2}=17$ for the renormalization scale. The curves correspond to the best-fit parameters obtained fitting only $\beta=2.0$, $\beta=2.2$ and $\beta=2.3$ (subset $S_{2}$ ) and are drawn for the corresponding lattice spacing. The black curve indicates the continuum results.

constants $F$ and $B$ on the choice of renormalization scale $\left(w_{0}^{\chi} p\right)^{2}$. The main result in the text are obtained using $\left(w_{0}^{\chi} p\right)^{2}=7$ as the reference momentum scale. Here we present the same analysis for another value of the reference momentum scale: $\left(w_{0}^{\chi} p\right)^{2}=17$. This corresponds to a much higher scale where lattice cutoff effects are expected

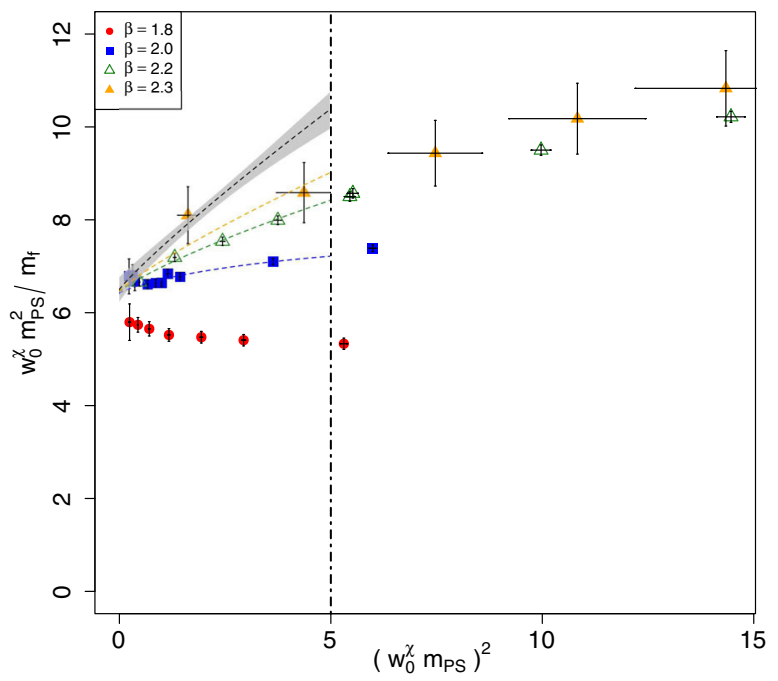

FIG. 15. $m_{\mathrm{PS}}^{2} / m_{\mathrm{f}}$ versus $m_{\mathrm{PS}}^{2}$ for four lattice spacings, using $\left(w_{0}^{\chi} p\right)^{2}=17$ for the renormalization scale. The curves correspond to the best-fit parameters obtained fitting only $\beta=2.0$, $\beta=2.2$ and $\beta=2.3$ (subset $S_{2}$ ) and are drawn for the corresponding lattice spacing. The black curve indicates the continuum results.

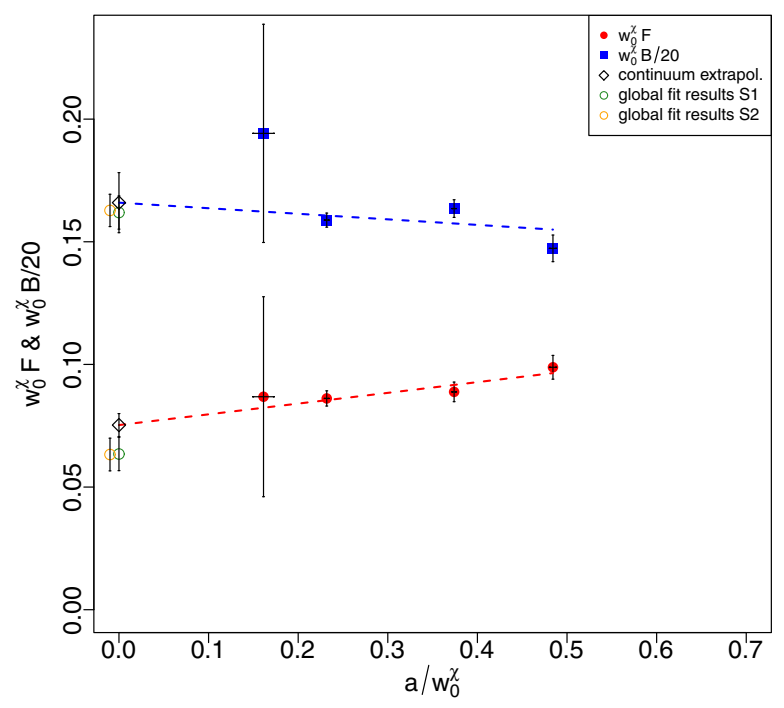

FIG. 16. Analogue of Fig. 11 obtained for reference momentum $\left(w_{0}^{\chi} p\right)^{2}=17$.

to become more relevant. We show below in Figs. 14,15 and 16 the analysis of $m_{\mathrm{PS}}, f p s$ and the scaling plot $F$ and $B$ using $\left(w_{0}^{\chi} p\right)^{2}=17$. The corresponding results for the chiral parameters read $w_{0}^{\chi} B=3.32(24)(8)$ and $w_{0}^{\chi} F=0.075(5)(12)$. Setting the scale to be $F=$ $246 \mathrm{GeV}$, one thus deduces $w_{0}^{x}=6.0(4)(9) \times 10^{-5} \mathrm{fm}$. The value of the condensate then reads $\Sigma^{1 / 3} / F=$ 4.48(28) (statistical and systematical errors have been combined). Although the dependence on the reference scale is clear at finite lattice spacing, the continuum extrapolated results are almost insensitive to this choice within our errors and they are therefore in agreement with the ones obtained in the main text.

\section{APPENDIX C: TOPOLOGY}

Besides being an efficient way of setting the scale, fields smoothed at nonzero flow time allow for a convenient definition of the topological charge, in terms of the straightforward discretization of the topological charge density.

We plot in Fig. 17 the topological charge as a function of the Monte Carlo time for two $\beta$ values at the lightest quark mass for a fixed value of $c=\sqrt{8 t} / L \approx 0.5$. In general, we observe that the average topological charge is compatible with zero for all our runs and that the fluctuations decrease with the fermion mass, as expected. Even if we observe larger correlation times for the topological charge at smaller quark masses, our simulations still explore all topological sectors with a good efficiency. The corresponding distribution of the topological charge is approximately Gaussian as shown in Fig. 18 . 

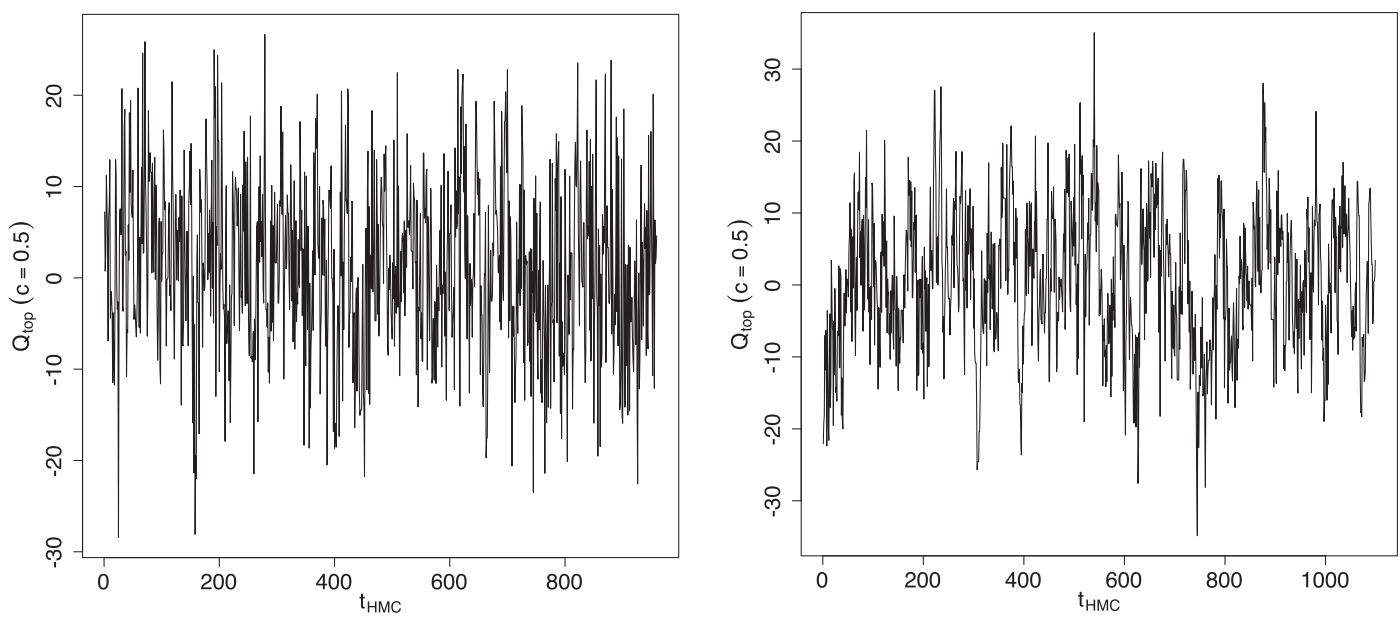

FIG. 17. History of the topological charge for the most chiral run at $\beta=2.0$ (left) and $\beta=2.2$ (right) as a function of the Monte Carlo time $t_{\mathrm{HMC}}$.
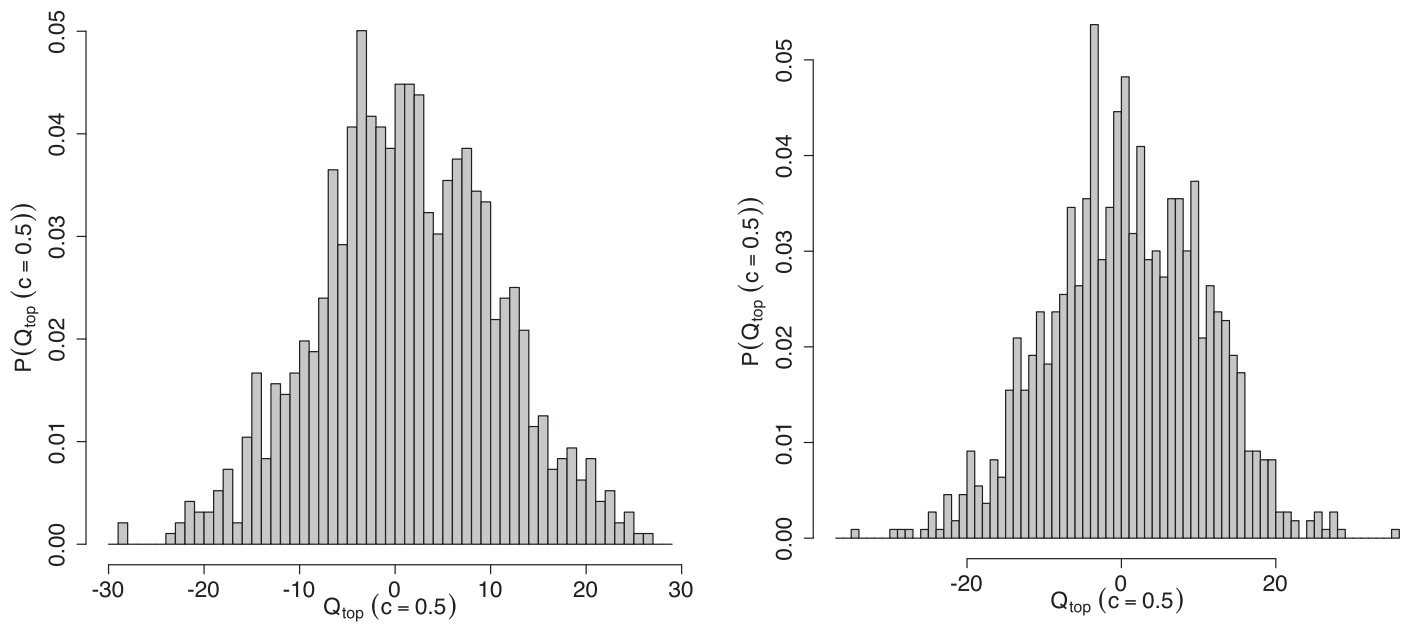

FIG. 18. Histogram of the topological charge for the same run as in Fig. 17 for $\beta=2.0$ (left) and $\beta=2.2$ (right).

[1] A. Hietanen, R. Lewis, C. Pica, and F. Sannino, Fundamental composite Higgs dynamics on the lattice: SU(2) with two flavors, J. High Energy Phys. 07 (2014) 116.

[2] E. Molinaro, F. Sannino, and N. Vignaroli, Minimal composite dynamics versus axion origin of the diphoton excess, Mod. Phys. Lett. A 31, 1650155 (2016).

[3] S. Matsuzaki and K. Yamawaki, $750 \mathrm{GeV}$ diphoton signal from one-family walking technipion, Mod. Phys. Lett. A 31, 1630016 (2016).

[4] D. B. Franzosi and M. T. Frandsen, Symmetries and composite dynamics for the $750 \mathrm{GeV}$ diphoton excess, arXiv:1601.05357.

[5] H. S. Fukano, M. Kurachi, S. Matsuzaki, K. Terashi, and K. Yamawaki, $2 \mathrm{TeV}$ walking technirho at LHC?, Phys. Lett. B 750. 259 (2015).
[6] D. B. Franzosi, M. T. Frandsen, and F. Sannino, Diboson signals via Fermi scale spin-one states, Phys. Rev. D 92, 115005 (2015).

[7] CMS Collaboration, Search for new physics in high mass diphoton events in proton-proton collisions at $13 \mathrm{TeV}$, Report No. CMS-PAS-EXO-15-004.

[8] ATLAS Collaboration, Search for resonances decaying to photon pairs in $3.2 \mathrm{fb}^{-1}$ of $p p$ collisions at $\sqrt{s}=13 \mathrm{TeV}$ with the ATLAS detector, Report No. ATLAS-CONF-2015-081.

[9] G. Aad et al. (ATLAS collaboration), Search for high-mass diboson resonances with boson-tagged jets in proton-proton collisions at $\sqrt{s}=8 \mathrm{TeV}$ with the ATLAS detector, J. High Energy Phys. 12 (2015) 055.

[10] S. Weinberg, Implications of dynamical symmetry breaking, Phys. Rev. D 13, 974 (1976). 
[11] L. Susskind, Dynamics of spontaneous symmetry breaking in the Weinberg-Salam theory, Phys. Rev. D 20, 2619 (1979).

[12] D. B. Kaplan and H. Georgi, SU(2) $\times \mathrm{U}(1)$ breaking by vacuum misalignment, Phys. Lett. 136B, 183 (1984).

[13] D. B. Kaplan, H. Georgi, and S. Dimopoulos, Composite Higgs scalars, Phys. Lett. 136B, 187 (1984).

[14] F. Sannino and J. Schechter, Chiral phase transition for $\mathrm{SU}(\mathrm{N})$ gauge theories via an effective Lagrangian approach, Phys. Rev. D 60, 056004 (1999).

[15] D. K. Hong, S. D. H. Hsu, and F. Sannino, Composite Higgs from higher representations, Phys. Lett. B 597, 89 (2004).

[16] D. D. Dietrich, F. Sannino, and K. Tuominen, Light composite Higgs from higher representations versus electroweak precision measurements: Predictions for CERN LHC, Phys. Rev. D 72, 055001 (2005).

[17] D. D. Dietrich, F. Sannino, and K. Tuominen, Light composite Higgs and precision electroweak measurements on the $\mathrm{Z}$ resonance: An update, Phys. Rev. D 73, 037701 (2006).

[18] F. Sannino, Conformal dynamics for $\mathrm{TeV}$ physics and cosmology, Acta Phys. Pol. B 40, 3533 (2009).

[19] D. D. Dietrich and F. Sannino, Conformal window of SU(N) gauge theories with fermions in higher dimensional representations, Phys. Rev. D 75, 085018 (2007).

[20] R. Foadi, M. T. Frandsen, and F. Sannino, 125 GeV Higgs boson from a not so light technicolor scalar, Phys. Rev. D 87, 095001 (2013)

[21] T. Appelquist, P. S. Rodrigues da Silva, and F. Sannino, Enhanced global symmetries and the chiral phase transition, Phys. Rev. D 60, 116007 (1999).

[22] T. A. Ryttov and F. Sannino, Ultra minimal technicolor and its dark matter TIMP, Phys. Rev. D 78, 115010 (2008).

[23] J. Galloway, J. A. Evans, M. A. Luty, and R. A. Tacchi, Minimal conformal technicolor and precision electroweak tests, J. High Energy Phys. 10 (2010) 086.

[24] G. Cacciapaglia and F. Sannino, Fundamental composite (Goldstone) Higgs dynamics, J. High Energy Phys. 04 (2014) 111.

[25] T. Appelquist, Z.-y. Duan, and F. Sannino, Phases of chiral gauge theories, Phys. Rev. D 61 (2000) 125009.

[26] Y.-L. Shi and R. Shrock, Renormalization-group evolution of chiral gauge theories, Phys. Rev. D 91, 045004 (2015).

[27] Y.-L. Shi and R. Shrock, $A_{k} \bar{F}$ chiral gauge theories, Phys. Rev. D 92, 105032 (2015).

[28] G. Cacciapaglia and F. Sannino, An ultraviolet chiral theory of the top for the fundamental composite (Goldstone) Higgs, Phys. Lett. B 755, 328 (2016).

[29] S. Nussinov, Technocosmology: Could a technibaryon excess provide a "natural", missing mass candidate?, Phys. Lett. 165B, 55 (1985).

[30] S. M. Barr, R. S. Chivukula, and E. Farhi, Electroweak fermion number violation and the production of stable particles in the early Universe, Phys. Lett. B 241, 387 (1990).

[31] S. B. Gudnason, C. Kouvaris, and F. Sannino, Towards working technicolor: Effective theories and dark matter, Phys. Rev. D 73, 115003 (2006).

[32] S. B. Gudnason, C. Kouvaris, and F. Sannino, Dark matter from new technicolor theories, Phys. Rev. D 74, 095008 (2006).
[33] M. T. Frandsen, S. Sarkar, and K. Schmidt-Hoberg, Light asymmetric dark matter from new strong dynamics, Phys. Rev. D 84, 051703 (2011).

[34] Y. Hochberg, E. Kuflik, T. Volansky, and J. G. Wacker, Mechanism for thermal relic dark matter of strongly interacting massive particles, Phys. Rev. Lett. 113, 171301 (2014).

[35] E. D. Carlson, M. E. Machacek, and L. J. Hall, Selfinteracting dark matter, Astrophys. J. 398, 43 (1992).

[36] A. A. de Laix, R. J. Scherrer, and R. K. Schaefer, Constraints of self-interacting dark matter, Astrophys. J. 452, 495 (1995).

[37] Y. Hochberg, E. Kuflik, H. Murayama, T. Volansky, and J. G. Wacker, Model for thermal relic dark matter of strongly interacting massive particles, Phys. Rev. Lett. 115, 021301 (2015).

[38] M. Hansen, K. Langble, and F. Sannino, SIMP model at NNLO in chiral perturbation theory, Phys. Rev. D 92, 075036 (2015).

[39] J. Wess and B. Zumino, Consequences of anomalous Ward identities, Phys. Lett. 37B, 95 (1971).

[40] E. Witten, Global aspects of current algebra, Nucl. Phys. B223, 422 (1983).

[41] E. Witten, Current algebra, baryons, and quark confinement, Nucl. Phys. B223, 433 (1983).

[42] Y. Hochberg, E. Kuflik, and H. Murayama, SIMP spectroscopy, J. High Energy Phys. 05 (2016) 090.

[43] S.-M. Choi and H. M. Lee, Resonant SIMP dark matter, Phys. Lett. B 758, 47 (2016).

[44] R. Lewis, C. Pica, and F. Sannino, Light asymmetric dark matter on the lattice: $S U(2)$ technicolor with two fundamental flavors, Phys. Rev. D 85, 014504 (2012).

[45] R. Arthur, V. Drach, M. Hansen, A. Hietanen, C. Pica, and F. Sannino, Scattering lengths in SU(2) gauge theory with two fundamental fermions, Proc. Sci., LATTICE2014 (2014) 271, arXiv:1412.4771.

[46] A. Hietanen, R. Lewis, C. Pica, and F. Sannino, Composite Goldstone dark matter: Experimental predictions from the lattice, J. High Energy Phys. 12 (2014) 130.

[47] V. Drach, A. Hietanen, C. Pica, J. Rantaharju, and F. Sannino, Template composite dark matter: $\mathrm{SU}(2)$ gauge theory with 2 fundamental flavours, Proc. Sci., LATTICE2015 (2015) 234.

[48] M. Hayakawa, K. I. Ishikawa, S. Takeda, M. Tomii, and N. Yamada, Lattice Study on quantum-mechanical dynamics of two-color QCD with six light flavors, Phys. Rev. D 88, 094506 (2013).

[49] A. Amato, T. Rantalaiho, K. Rummukainen, K. Tuominen, and S. Thtinen, Approaching the conformal window: systematic study of the particle spectrum in SU(2) field theory with $N_{f}=2,4$ and 6, Proc. Sci., LATTICE2015 (2015) 225, arXiv:1511.04947.

[50] L. Del Debbio, A. Patella, and C. Pica, Higher representations on the lattice: Numerical simulations. SU(2) with adjoint fermions, Phys. Rev. D 81, 094503 (2010).

[51] P. A. Boyle, A. Juttner, C. Kelly, and R. D. Kenway, Use of stochastic sources for the lattice determination of light quark physics, J. High Energy Phys. 08 (2008) 086.

[52] L. Del Debbio, L. Giusti, M. Luscher, R. Petronzio, and N. Tantalo, QCD with light Wilson quarks on fine lattices. II. 
DD-HMC simulations and data analysis, J. High Energy Phys. 02 (2007) 082.

[53] F. Bursa, L. Del Debbio, D. Henty, E. Kerrane, B. Lucini, A. Patella, C. Pica, T. Pickup, and A. Rago, Improved lattice spectroscopy of minimal walking technicolor, Phys. Rev. D 84, 034506 (2011).

[54] M. Lscher, Properties and uses of the Wilson flow in lattice QCD, J. High Energy Phys. 08 (2010) 071.

[55] M. Luscher and P. Weisz, Perturbative analysis of the gradient flow in non-Abelian gauge theories, J. High Energy Phys. 02 (2011) 051.

[56] S. Borsanyi et al., High-precision scale setting in lattice QCD, J. High Energy Phys. 09 (2012) 010.

[57] O. Bar and M. Golterman, Chiral perturbation theory for gradient flow observables, Phys. Rev. D 89, 034505 (2014).

[58] G. Martinelli, C. Pittori, C. T. Sachrajda, M. Testa, and A. Vladikas, A General method for nonperturbative renormalization of lattice operators, Nucl. Phys. B445, 81 (1995).
[59] M. Gockeler, R. Horsley, H. Oelrich, H. Perlt, D. Petters, P.E.L. Rakow, A. Schäfer, G. Schierholz, and A. Schiller, Nonperturbative renormalization of composite operators in lattice QCD, Nucl. Phys. B544, 699 (1999).

[60] J. C. Vink and U.-J. Wiese, Gauge fixing on the lattice without ambiguity, Phys. Lett. B 289, 122 (1992).

[61] P. F. Bedaque, Aharonov-Bohm effect and nucleon nucleon phase shifts on the lattice, Phys. Lett. B 593, 82 (2004).

[62] C. T. Sachrajda and G. Villadoro, Twisted boundary conditions in lattice simulations, Phys. Lett. B 609, 73 (2005).

[63] J.-R. Cudell, A. Le Yaouanc, and C. Pittori, Pseudoscalar vertex, Goldstone boson and quark masses on the lattice, Phys. Lett. B 454, 105 (1999).

[64] J. R. Cudell, A. Le Yaouanc, and C. Pittori, Large pion pole in $Z_{S}^{\mathrm{MOM}} / Z_{P}^{\mathrm{MOM}}$ from from Wilson action data, Phys. Lett. B 516, 92 (2001).

[65] J. Bijnens and J. Lu, Technicolor and other QCD-like theories at next-to-next-to-leading order, J. High Energy Phys. 11 (2009) 116. 